\title{
High-Precision Atomic Mass Measurements for Fundamental Constants
}

\author{
Edmund G. Myers \\ Department of Physics, Florida State University, Tallahassee, FL 32306-4350, USA; emyers@fsu.edu
}

Received: 4 January 2019; Accepted: 28 February 2019; Published: 18 March 2019

\begin{abstract}
Atomic mass measurements are essential for obtaining several of the fundamental constants. The most precise atomic mass measurements, at the $10^{-10}$ level of precision or better, employ measurements of cyclotron frequencies of single ions in Penning traps. We discuss the relation of atomic masses to fundamental constants in the context of the revised SI. We then review experimental methods, and the current status of measurements of the masses of the electron, proton, neutron, deuteron, tritium, helium-3, helium-4, oxygen-16, silicon-28, rubidium-87, and cesium-133. We conclude with directions for future work.
\end{abstract}

Keywords: atomic mass; fundamental constants; precision measurement; Penning trap

\section{Introduction}

The fundamental constants enable the quantitative unification of physical science. At the highest level of precision, values for dimensionless constants such as the fine-structure constant, obtained from different types of measurement, can be used to test the validity of fundamental theory and search for physics beyond the so-called Standard Model [1]. While the atomic masses of the electron, the isotopes of hydrogen and helium, and the neutron are themselves regarded as fundamental constants, others are necessary for various routes to obtaining other constants. Here we discuss precision atomic mass measurements that are relevant to the determination of fundamental constants as in the Committee on Data for Science and Technology (CODATA) evaluations [2,3]. Our aim is not to attempt any rigorous analysis of the relevant data as is done by the authors of the comprehensive Atomic Mass Evaluations (AME), (e.g., see references $[4,5])$, but to enable the reader to see those measurements that have the greatest impact. A previous review of precision atomic mass measurements can be found in reference [6]. Other related reviews, which also discuss applications to nuclear physics are references [7-10].

After some remarks about the unified atomic mass unit " $\mathrm{u}$ " and its relation to the "New-SI" $\mathrm{kg}$, we review current techniques for precision atomic mass measurements. These generally involve measuring cyclotron frequency ratios of ions in Penning ion traps. As well as these methods, we outline the main sources of measurement uncertainty. We then discuss mass measurements of the "light ions", i.e., the isotopes of hydrogen and helium; and then of the alkali metal atoms ${ }^{87} \mathrm{Rb}$ and ${ }^{133} \mathrm{Cs}$, which are important for the atom-recoil method for the fine-structure constant. Next, we discuss the special case of the atomic mass of the electron, which has now been most accurately obtained from the electronic $g$-factor (Zeeman effect) of hydrogen-like ions. Here, and for related $g$-factor measurements, the atomic mass of the ion is also needed. We then briefly consider atomic masses relating to Avogadro's number and the Boltzmann constant. We conclude by indicating where future atomic mass measurements are needed. 


\subsection{Relative Atomic Masses, the Unified Atomic Mass Unit (u) and Dalton (Da)}

The most precise measurements of the masses of atoms are obtained using Penning ion traps, which yield mass ratios of ions from (inverse) cyclotron frequency ratios (CFRs). The mass ratios of ions, including molecular ions, can be corrected to give mass ratios of atoms by allowing for the mass of the missing electrons and the "energies of formation" (EOF) of the ions from their constituent atoms, (e.g., see references $[2,11])$. In the case of monatomic ions, the EOFs are simply ionization energies, while in the case of molecular ions they must also include the binding energy of the molecule, (e.g., see reference [12]). For low charge-states of all atoms, all charge-states of lighter atoms, and for simple molecular ions in known ro-vibrational states, this can usually be done without any significant loss of precision. If the mass of the atom (or other particle) can then be related to the mass of an atom of ${ }^{12} \mathrm{C}$ (either through a single CFR, or sometimes, through a chain, or even an intricate web of related CFRs) then the atom's mass can be expressed in so-called "unified atomic mass units", with symbol u, which is defined as $1 / 12$ of the mass of a free atom of ${ }^{12} \mathrm{C}$ at rest. This is what is meant by (relative) "atomic mass" (it is the mass of a particle, which may or may not be an atom, expressed in $u$ ). While physicists favor $\mathrm{u}$, chemists generally use the name dalton (Da) for the identical unit. The $\mathrm{u}$ is also distinct from the older and deprecated amu, which was based on the mass of ${ }^{16} \mathrm{O}$ [4], although this is not always recognized in the literature. It should also be appreciated that relating masses of atoms to the $\mathrm{u}$, although conventional, is not always necessary. For instance, as regards the determination of the Rydberg from spectroscopy of hydrogen, what matters is the mass ratio of the electron to the proton. As a point of notation, in this article we use upper case, e.g., $M[e]$ or $M\left[{ }^{3} \mathrm{He}\right]$ to denote (relative) masses expressed in $\mathrm{u}$, and lower case, e.g., $m_{e}$, when this distinction is not needed.

\subsection{Relation of $u$ to the Kilogram in the New SI}

In late 2018, it was agreed by the international General Conference on Weights and Measures, that from May 2019, the base units of the International System of Units (SI) were to be redefined by adopting fixed values for the Planck constant $h$, the elementary electric charge $e$, the Boltzmann constant $k_{B}$, and the Avogadro constant $N_{A}$. The speed of light in vacuum $c$ was already a fixed constant. Given that the second is defined in terms of the frequency of the hyperfine transition in the ground state of the ${ }^{133} \mathrm{Cs}$ atom, it follows (Special Relativity and Quantum Mechanics being assumed) that the $\mathrm{kg}$ is now defined by the fixed $h$, the ampere by the fixed $e$, the kelvin by the fixed $k_{B}$, and the mole by the fixed $N_{A}$. A general consequence is the conceptual shift that measurements (often chains of measurements), that formerly measured these fundamental constants, no longer do that. After the redefinition, these measurements are just as important, but now have the role of realizing the SI base units from the fundamental constants. An example of this is the Kibble (or Watt) balance [13], which effectively compares the mechanical and electrical watt, the latter being related to $h$ using the Josephson effect and the quantized Hall effect. Formerly the Kibble balance measured $h$ relative to the artifact $\mathrm{kg}$; now it realizes the $\mathrm{kg}$ from the defined $h$. Because this can now be done reproducibly at the few $10^{-8}$ level, the re-defined $\mathrm{kg}$ is more stable and reproducible than the platinum-iridium alloy artifact $\mathrm{kg}$ standard adopted in 1889 .

Another related example, and where atomic masses are involved, is the X-Ray-Crystal-Density (XRCD) technique for determining the number of atoms in a near-perfect spherical crystal of silicon, by precision $x$-ray measurements of the crystal's lattice spacing, combined with optical measurements of the crystal's diameter, (e.g., see reference [14]). Formerly, after measuring the crystal's mass against the artifact SI kg, and knowing the atomic mass of $\mathrm{Si}$ [15], this related $\mathrm{u}$ to the $\mathrm{kg}$, and equivalently, measured the Avogadro constant $N_{A}$, at the few $10^{-8}$ level. Now, with $N_{A}$ fixed, $N_{A}$ no longer relates $\mathrm{u}$ to the $\mathrm{kg}$ (and, from the physicist's point of view, has lost significance). The "atom counting" of the $\mathrm{XRCD}$ technique still relates the atomic mass unit $\mathrm{u}$ to a macroscopic mass. However, since $\mathrm{u}$ can now be related to the redefined-SI $\mathrm{kg}$ in other and more precise ways, see below, this procedure is now an alternative way of realizing the macroscopic $\mathrm{kg}$ (also at the few $10^{-8}$ level). 
In the new-SI with a defined $h$, the $\mathrm{u}$ and the $\mathrm{kg}$ can be accurately related using atom-recoil measurements that yield $h / m_{\text {atom }}$ combined with atomic mass measurements of the particular atom relative to ${ }^{12} \mathrm{C}$ (e.g., see [16]). Atom recoil measurements are further discussed in relation to the fine-structure constant in Section 4. One can also use the relation between the Rydberg constant $R_{\infty}$, the fine-structure constant $\alpha$, and the absolute electron mass $m_{e}$,

$$
2 h R_{\infty}=\alpha^{2} m_{e} c .
$$

In this expression, the least well-known quantity is $\alpha$, which in CODATA14 has a fractional uncertainty of $2.3 \times 10^{-10}$, while $R_{\infty}$ has a fractional uncertainty of $5.9 \times 10^{-12}$ [2]. Thus, $m_{e}$ is determined relative to the SI-kg essentially with the same precision as $\alpha^{2}$. In addition, $M[e]$, the mass of the electron relative to ${ }^{12} \mathrm{C}$, has now been experimentally determined with an uncertainty of $2.9 \times 10^{-11}$ [17] (see Section 5), also significantly less than the uncertainty of $\alpha^{2}$. Hence, $\mathrm{u}$ can be related to the new-SI $\mathrm{kg}$ with a relative uncertainty equivalent to that of $\alpha^{2}$, i.e., to better than $5 \times 10^{-10}$, an improvement of more than 20 compared to its relationship to the "old-SI" $\mathrm{kg}$. (Using the new-SI value for $h$ [3], and CODATA14 values for $R_{\infty}, \alpha$ and $M[e]$, one obtains $\mathrm{u}=1.660539067$ $95(78) \times 10^{-27} \mathrm{~kg}$, c.f. the CODATA14 value, $\mathrm{u}=1.660539040(20) 10^{-27} \mathrm{~kg}$ ). Since this is substantially better than what can be obtained from the XRCD technique, it follows that the XRCD technique now has the practical role of providing an alternative to the Kibble balance in realizing the new-SI kg from its definition.

\section{Precision Measurement of Atomic Masses Using Penning Ion Traps}

\subsection{The Precision Penning Trap}

A Penning trap consists of a set of cylindrically symmetric electrodes, a central ring, and two end-caps, immersed in a uniform magnetic field. In a precision trap [18], extra field-compensation electrodes are placed between the ring and endcaps to adjust the shape of the potential, and the uniform magnetic field is produced by a carefully shimmed superconducting magnet. In some Penning traps, the electrodes have hyperboloidal inner surfaces, while in others the electrodes, including those that function as the endcaps, consist of circular tubes. This "open endcap" design facilitates moving ions between adjacent traps and the introduction of microwave or laser radiation. The electrostatic field is produced by applying voltages to the electrodes, typically $<100 \mathrm{~V}$, using high stability voltage sources through low-pass filters.

In the ideal Penning trap the electrostatic potential $V(\rho, z)$ has the purely quadratic form given (in cylindrical co-ordinates) by $V(\rho, z)=\left(V_{0} / 2 d^{2}\right)\left(z^{2}-\rho^{2} / 2\right)$, where the $z$-axis is aligned with the uniform magnetic field, $V_{0}$ is the voltage between the ring and endcaps and $d$ is a parameter characterizing the trap size. For the hyperboloidal electrode trap, $2 d^{2}=z_{0}{ }^{2}+\rho_{0}{ }^{2} / 2$, where $2 z_{0}$ is the shortest distance between the endcaps and $2 \rho_{0}$ is the smallest internal diameter of the ring. The motion of a single trapped ion is the superposition of a simple harmonic motion along the $z$-axis (called the axial motion, at frequency $f_{z}$ ), and two circular motions, the modified (by the electrostatic potential) cyclotron motion at frequency $f_{c}{ }^{\prime}$, and the magnetron motion, which is a lower frequency motion of the ion about the center of the trap-and thus provides the radial confinement-at frequency $f_{m}$. The "true" cyclotron frequency, which in a magnetic field of magnitude $B$ without any electrostatic field is given by $f_{c}=(1 / 2 \pi) q B / m$, is now given by a relation involving all three measurable frequencies, $f_{c}{ }^{2}=f_{c}{ }^{2}+$ $f_{z}^{2}+f_{m}^{2}$.

Remarkably, in the limit of small amplitudes of the motion, this relation is still true in a trap where the electrostatic potential is not perfectly cylindrically symmetric, and is not perfectly aligned with the magnetic field, where $f_{c}{ }^{\prime}, f_{z}$, and $f_{m}$ are now the frequencies of the motions that approximate those in the ideal trap. This is known as the Invariance Theorem [18]. Hence, if all three mode frequencies are measured, $f_{c}{ }^{2}=f_{c}{ }^{2}+f_{z}{ }^{2}+f_{m}{ }^{2}$ provides a robust prescription for obtaining the "true" cyclotron frequency, which is inversely proportional to the mass of the ion. Further, by arranging that $f_{z}<<$ 
$f_{c^{\prime}}, f_{m}$ can be obtained from $f_{m}=\left(f_{z}{ }^{2} / 2 f_{c}{ }^{\prime}\right)(1+\delta)$, where $\delta$, which is typically $<<10^{-3}$, can be used to parameterize the combined effect of the ellipticity of the electrostatic field and its tilt with respect to the magnetic field. Because of the frequency hierarchy, the absolute uncertainties of $f_{z}$ and $f_{m}$ that can be allowed without compromising the measurement of $f_{c}$ are greater than that of $f_{c}{ }^{\prime}$ by factors of $f_{c} / f_{z}$ and $f_{c} / f_{m}$, respectively. Hence, while in order to increase statistical precision it is necessary to repeat measurements of $f_{c}{ }^{\prime}$ and $f_{z}$, measurements of $f_{m}$ are only needed to obtain and check the stability of $\delta$. In Penning traps used for the most precise measurements, the chamber housing the electrodes is maintained at close to LHe temperature, resulting in extreme $\left(<<10^{-15} \mathrm{mbar}\right)$ high-vacuum [19]. Hence single ions can be trapped and manipulated, and measurements made on them for many days.

\subsection{Measuring the Cyclotron Frequency}

\subsubsection{Detecting the Axial Motion}

In cryogenic Penning traps the axial motion of a single ion can be detected, and also damped, by bringing it into resonance with a high-quality-factor tank circuit, whose main component is a low-loss inductor, i.e., a coil, often superconducting, connected across the trap electrodes. The oscillating ion induces oscillating image charges on the trap electrodes, and hence a current through the detection circuit. Due to the high-impedance of the detection circuit at its resonance frequency, this results in a significant oscillatory voltage that back-acts on the ion, damping its motion till it comes to thermal equilibrium with the coil, which is typically at close to $4.2 \mathrm{~K}$. The coil is electrically coupled to an amplifier consisting of an FET transistor, (e.g., see references $[18,20]$ ) or else a dc-SQUID [21]. The axial frequency can hence be measured by Fourier-transforming the amplified signal due to the axial motion of the ion. This motion can be the damped "ring-down" after the ion has been axially excited by an rf-drive pulse. Alternatively, since an unexcited ion partially cancels the Johnson noise from the resonant circuit at the ion's axial frequency, the axial frequency can be determined from the resulting dip in the noise spectrum [18].

\subsubsection{Swept Continuous Cyclotron Drive with Axial Frequency Shift Detection}

Although the cyclotron motion can also be directly detected and damped using image current techniques, (e.g., see references [22,23]), for a single ion the 1/e damping times are typically several minutes and thus inconveniently long. More often, the radial modes are detected by coupling them to the axial mode. In the case of the technique used by the Penning trap group at the University of Washington (UW) [24], $f_{c}{ }^{\prime}$ was measured by making use of the shift in axial frequency that occurs when the cyclotron radius changes in an imperfectly homogeneous magnetic field. Hence, as a weak $\mathrm{rf}$ drive is swept through $f_{c}{ }^{\prime}$, either from below or from above, the excitation of the cyclotron motion is signaled by a shift in axial frequency as the drive frequency passes through $f_{c}{ }^{\prime}$. The best estimate of $f_{c}{ }^{\prime}$ is obtained by interpolating between the two corner frequencies corresponding to the different directions of the scan. Disadvantages of this method are the requirements for high magnetic field and trap voltage stability, and that the responses to the up and down scans are not symmetrical and depend on various instrumental settings [25].

\subsubsection{Double Dip (Avoided Crossing) Technique}

The radial and axial modes of motion can be resonantly coupled by applying a "tilted" quadrupolar rf electric field at the frequency $f_{c}{ }^{\prime}-f_{z}$ for the cyclotron mode (and $f_{z}+f_{m}$ for the magnetron mode) [26]. If the rf drive is applied continuously at $f_{c}{ }^{\prime}-f_{z}$, the "action" oscillates between the cyclotron and axial modes at a frequency proportional to the coupling strength, usually called the Rabi frequency. The Fourier transform of the axial signal is then split into two components, separated by the Rabi frequency and symmetric about the original axial frequency. This enables $f_{c}{ }^{\prime}$ to be determined from either the double peaks after pulsed excitation of the axial motion [26], or, 
by detecting a "double dip" in the noise spectrum, (e.g., see reference [27]). Similar methods can be used to measure $f_{m}$.

\subsubsection{Pulse-and-Phase and Pulse-and-Amplify Techniques}

The so-called "Pulse-and-Phase" (PnP) technique was first developed by the atomic mass measurement group at the Massachusetts Institute of Technology (MIT) [26,28], and has since been used extensively at Florida State University (FSU), where the MIT Penning trap was relocated in 2003, (e.g., see references $[15,29-32])$. Here, after centering the ion by applying the coupling drives at $f_{c}{ }^{\prime}$ $-f_{z}$ and $f_{z}+f_{m}$, and allowing the axial motion to damp, a pulse is applied at a frequency close to $f_{c}^{\prime}$. This produces a cyclotron motion with a well-defined radius and phase. This is followed by a delay, $T_{\text {evol }}$ (the phase evolution time), during which the cyclotron motion continues, unperturbed, evolving phase. The final phase of the modified cyclotron motion is then "read out" by applying a "pi-pulse" (producing half a Rabi oscillation) at close to $f_{c}{ }^{\prime}-f_{z}$, which converts the cyclotron motion phase-coherently into axial motion. Thus, the final cyclotron phase $\varphi_{c^{\prime}}$ is mapped onto the axial motion, which is then detected. By repeating the process with different values of $T_{\text {evol }}, f_{c}{ }^{\prime}$ is obtained from $f_{c^{\prime}}=(1 / 2 \pi) d \varphi_{c^{\prime}} / d T_{\text {evol }}$. In the MIT/FSU trap, with a cyclotron frequency of $\sim 4 \mathrm{MHz}(\sim 40 \mathrm{MHz}), \varphi_{c^{\prime}}$ can be typically measured with an uncertainty $<1 / 10$ of a cycle after a maximum $T_{\text {evol }}=100 \mathrm{~s}(10 \mathrm{~s})$, corresponding to a fractional frequency resolution of $\sim 2.5 \times 10^{-10}$. However, because the phase must be "unwrapped", as many as $12 \mathrm{PnPs}$ with a range of $T_{\text {evol }}$ are often used to give a single measurement of $f_{c}{ }^{\prime}$ [26].

In the Pulse-and-Amplify (PnA) method [33], instead of the pi-pulse, the coupling drive is applied at the end of $T_{\text {evol }}$ as the sum frequency $f_{c}{ }^{\prime}+f_{z}$. As in the PnP, this maps the cyclotron phase onto the axial phase. However, it also produces parametric amplification of both modes and thus results in a larger axial signal, enabling measurements with smaller initial cyclotron radii (see Section 2.3.7).

\subsubsection{Cyclotron Frequency Measurement Using Time-of-Flight Detection}

An exception to the use of a cryogenic Penning trap with image charge detection is the work of the SMILETRAP group at the University of Stockholm, who used a room temperature trap connected to an external ion source to make cyclotron frequency measurements using the "time-of-flight" (TOF) detection technique [34], as is often used for nuclear physics [10]. In this method, an ion is injected into the trap and excited to a significant magnetron radius, after which a rf pulse is applied at close to the magnetron-to-cyclotron coupling frequency $f_{c}{ }^{\prime}+f_{m}$, which is approximately equal to the free-space cyclotron frequency $f_{c}$ [35]. Depending on the detuning of the drive from $f_{c}{ }^{\prime}+f_{m}$, this converts some of the magnetron motion to cyclotron motion. The ion is then ejected from the trap and detected in a micro-channel plate. Because the fringing field of the magnet exerts an axial force on the ion proportional to the cyclotron radius, the TOF measures the cyclotron radius and hence signals that the drive was on resonance. By repeating the process with many ions and different coupling frequencies, a resonance curve can be mapped out. The resolution of this procedure has been improved by using two pulses to produce Ramsey fringes [36-38], and, more recently, by using the so-called "phase imaging technique" [39], which is an ingenious adaptation of the PnP technique. However, disadvantages of these methods, compared to image-charge techniques, are that the motions of the ions in the Penning trap are relatively large and uncontrolled and that impurity ions perturb the measurements.

\subsection{Limitations to Precision Measurement of Cyclotron Frequency Ratios}

Here we discuss sources of uncertainty that limit the precision with which a cyclotron frequency ratio can be measured in a Penning trap, and some of the methods used to mitigate them. For definiteness, we focus on measurements using the pulse-and-phase technique, but similar considerations apply to other techniques. More details can be found in reference [6]. 


\subsubsection{Magnetic Field Variation}

Any variation of the magnetic field between measurements of $f_{c}$ of the two ions in a pair adds uncertainty to the measurement of the CFR. This can be addressed by careful engineering of the superconducting magnet to have high stability. For example, with a magnet with a fractional field drift $<2 \times 10^{-11} / \mathrm{h}$ [40], the UW group was able to take data with runs that consisted of repeated cyclotron frequency measurements on one ion for several days, followed by repeated measurements on the second ion, also for many days. With a less stable magnet, it is desirable to alternate between measurements on the two ions as frequently as possible. At MIT, a semi-automated procedure for making and remaking ions within the trap ("make and remake technique") was developed, sometimes allowing up to 8 pairs of measurements in a single $4 \mathrm{~h}$ run [41]. This procedure was initially adopted at FSU $[29,30]$. However, subsequently at FSU the "ion swapping" was greatly facilitated by trapping the two ions simultaneously, and alternating them between the center of the trap, where $f_{c}$ is measured using the PnP technique, and a large radius cyclotron "parking" orbit [22,42]. Alternatively, as developed at MIT in 2002, if the two ions have the same charge, and have masses with a fractional difference less than about $10^{-3}$, it can be arranged that the two ions orbit the center of the trap, 180 degrees apart, in a coupled magnetron orbit [43]. In the FSU technique, the measurements of $f_{c}$ are still alternate, with a delay of about 5 minutes between them due to the time taken to re-center the outer ion. In the MIT method, the measurements are truly simultaneous, thus the variation in the magnetic field can be almost completely cancelled. Another method, which has been developed by a collaboration of MPIK-Heidelberg, University of Mainz, GSI, and RIKEN (MPIK* for short), is to use multiple adjacent traps aligned along the axis of the same superconducting magnet $[27,44-46]$. Each ion is alternately shuttled from its own storage trap into the measurement trap where $f_{c}$ is measured. This scheme can be extended by having a third ion in an additional trap, which simultaneously monitors the magnetic field, or, by having two nearly identical measurement traps and swapping the ions to be compared between them, also with simultaneous measurement of their cyclotron frequencies [21,47].

\subsubsection{Amplitude-Dependent Shifts Due to Field Imperfections and Special Relativity}

Other limiting sources of error in measurements of a CFR are the shifts in $f_{c}{ }^{\prime}$ and $f_{z}$ due to magnetic and electrostatic field imperfections, and special relativity, combined with the finite amplitude of the axial motion, $a_{z}$, and the radii of the cyclotron and magnetron motions, $\rho_{c}$ and $\rho_{m}$. As discussed in detail in reference $[18,48-50]$, the trap field imperfections can be quantified using the coefficients $C_{n}$ of the expansion of the electrostatic potential in Legendre polynomials, and the coefficients $B_{n}$ of the expansion of the magnetic field in associated Legendre polynomials. Of these, the most important are the lowest order even terms, $C_{4}, C_{6}$, and $B_{2}$, which lead to shifts that depend on even powers of the mode amplitudes. (The odd-order terms can produce frequency shifts proportional to $B_{1}{ }^{2}, B_{1} C_{3}$, etc., but none that are first order in the coefficients.) For example, due to $C_{4}, B_{2}$, and special relativity, $f_{c}{ }^{\prime}$ is shifted according to

$$
\Delta f_{c}^{\prime} f_{c}^{\prime} \approx-(3 / 2)\left(f_{m} / f_{c}{ }^{\prime}\right)\left(C_{4} / C_{2}\right)\left(2 a_{z}{ }^{2}-\rho_{c}{ }^{2}-2 \rho_{m}{ }^{2}\right) / d^{2}+(1 / 2)\left(B_{2} / B_{0}\right)\left(a_{z}{ }^{2}-\rho_{c}{ }^{2}-\rho_{m}{ }^{2}\right)-(1 / 2)\left(2 \pi f_{c}{ }^{\prime} / c\right)^{2} \rho_{c}{ }^{2},
$$

where the three groups of terms correspond to the shifts due to electrostatic and magnetic imperfections, and special relativity, respectively. Fortunately, because the axial and cyclotron frequencies have different dependencies on the mode amplitudes, measurements of the variation of $f_{z}$ versus $\rho_{m}$ and $\rho_{c}$ can be used to independently measure the electrostatic and magnetic imperfections [48]. Further, the relativistic shift can be used for calibrating the cyclotron radius. Clearly, the strategy for reducing errors due to anharmonic shifts is to minimize the field imperfections by careful trap design and to use the smallest mode amplitudes possible during the measurement of $f_{c^{\prime}}$ and $f_{z}$. Equation (2) also shows that for a large $m / q$ ratio and thus low $f_{c}{ }^{\prime}$, electrostatic imperfections become more important. Conversely, for small $\mathrm{m} / \mathrm{q}$, that is for light ions and highly-charged ions, electrostatic imperfections are less important and special relativity becomes dominant. 


\subsubsection{Equilibrium Position Shifts}

In order to bring the two ions' axial motions into resonance with the narrow-band detection circuit, the trap voltage must be changed according to the change in $m / q$. In an ideal trap, this would not change the ion's average (or "equilibrium") position. However, due to construction imperfections, contact potentials and charge patches on the electrodes, symmetry is broken and small shifts in position do occur, both axially and radially, as the trap voltage is changed. Combined with gradients in the magnetic field, this leads to a shift in the CFR [21]. This effect can be quantified by measurements using different charge-states of the same atom.

\subsubsection{Image Charge Shifts}

The oscillating image charge the ion induces on the electrodes back-acts on the ion, hence perturbing all three mode frequencies. The fractional shift in cyclotron frequency it produces is proportional to the ion's mass and thus is independent of the charge state. Hence, this systematic shift cannot be measured by using different charge states. Instead, it must be calculated [51] or obtained by measurement of the magnetron frequency, or by using molecular ions. The contributions from the end-caps and ring vary as $1 / z_{0}{ }^{3}$ and as $1 / \rho_{0}{ }^{3}$, thus this systematic is reduced by using a larger trap.

\subsubsection{Shifts Due to Interaction with the Detector}

The back-action on the axial motion from the resonant detection circuit produces a shift to the axial frequency, which has a dispersion-like dependence on the detuning between the axial frequency and the detector resonance [21]. Consequently, the shift can be nulled by minimizing this detuning. Alternatively, given sufficient detector sensitivity, as is the case at the FSU trap using a dc-SQUID, the shift and its derivative with respect to detuning can be reduced by detecting the axial motion several detector resonance linewidths away from the detector resonance.

\subsubsection{Ion-Ion Interaction Shifts}

If two ions are simultaneously trapped in the same trap it is necessary to allow for ion-ion interaction. These shifts have been treated in detail in references [42,52]. In general, for the case of ions separated in the radial plane by a fixed distance $\rho_{s}$, the shifts can be separated into "ring-of-charge" effects, due to the modification to the time-averaged potential experienced by the ion whose cyclotron frequency is being measured; and "dynamical" effects, due to the coupling of the motions of the two ions. In the limit of small motional amplitudes of the ion being measured, the modification to the potential produced by the other ion satisfies the conditions of the Invariance Theorem. Thus, provided $f_{\mathcal{c}}{ }^{\prime}$ and $f_{z}$ are measured together, the derived $f_{c}$ is unaffected. However, the ring-of-charge does modify the effective $C_{4}$ and higher-order coefficients of the potential, leading to shifts that vary as $a_{z}{ }^{2} / \rho_{s}{ }^{5}$, etc. The dynamical effects can be considered as due to the measured ion inducing a small, non-resonant motion on the other ion, which then resonantly back-acts on the measured ion, shifting its mode frequencies. These shifts vary as $1 / \rho_{s}{ }^{6}$ and are inversely proportional to the detuning between the respective mode frequencies of the ions. Hence, they are a particular problem for an ion pair whose $\mathrm{m} / \mathrm{q}$ values are very close. In principle, ion-ion interaction effects can be made negligible by using a sufficiently large $\rho_{s}$. However, in the MIT two-ion technique, increased $\rho_{s}$ leads to a decoupling of the magnetron motion and to rapidly increasing anharmonic shifts to the CFR [48]. Therefore, the useful range of $\rho_{s}$ is limited.

\subsubsection{Detector Noise and Ion Temperature}

In the PnP technique, detector noise leads to uncertainty in the measurement of the phase of the axial motion after the pi-pulse, and hence in the determination of the final cyclotron phase. In addition, because of the initial (thermal) motion of the ion, which combines vectorially with the cyclotron motion impressed by the cyclotron drive pulse, there is variation in the ion's cyclotron phase at the start of 
$T_{\text {evol }}$, which also contributes jitter to the measured phase. Both of these "phase noise" effects limit the minimum cyclotron radius that can be used for the PnP measurement. The impressed cyclotron radius, combined with the trap imperfections and special relativity, then results in a systematic shift to the cyclotron frequency.

Further, the thermal cyclotron motion before the cyclotron drive pulse is applied also leads to variation, from $\mathrm{PnP}$ to $\mathrm{PnP}$, in the cyclotron radius, and hence in the anharmonic frequency shift during $T_{\text {evol }}$. This creates noise on $f_{c}{ }^{\prime}$ itself, and thus limits the maximum useful $T_{\text {evol }}$. Since the lowest-order anharmonic frequency shifts vary as $\rho_{c}{ }^{2}$, it follows that fluctuations in the thermal radius lead to frequency fluctuations approximately proportional to $\rho_{c}$ [48]. This then limits the maximum useful $\rho_{c}$. If cyclotron-to-axial coupling is used to cool the cyclotron motion, the resulting effective temperature is $\left(f_{c}^{\prime} / f_{z}\right) T_{z}$, where $T_{z}$ is the axial temperature [18]. Combined with the fact that relativistic shifts to the cyclotron frequency scale as $f_{c}{ }^{2}$, the initial cyclotron temperature is a particularly significant source of frequency noise for ions with small $\mathrm{m} / \mathrm{q}$.

One strategy for reducing the ion's initial cyclotron temperature (following cyclotron-to-axial coupling) is to first reduce the axial temperature by applying electronic feedback to the axial detector $[52,53]$. Feedback can also be applied to the ion's axial motion with minimal effect on the detector [54]. A greater reduction in the temperature of the cyclotron mode, that is to a few $\mathrm{K}$, can be achieved with a resonant circuit across the halves of a split ring electrode [22,23].

With a reduced thermal cyclotron radius, a reduced impressed cyclotron radius can then be used without excessive phase noise at the start of $T_{\text {evol }}$. The reduced impressed cyclotron radius then results in reduced anharmonic frequency shifts and fluctuations. However, with the PnP method, any reduction in total cyclotron radius is limited because, after the read-out cyclotron-to-axial coupling pulse, the resulting axial motion must be above the detector noise. Therefore, in this situation, the PnA method is especially advantageous. Starting with a small cyclotron radius, it can produce a large axial motion that produces a signal above the detector noise.

\subsubsection{Statistical and Systematic Errors}

Detector noise, fluctuations in the initial mode amplitudes combined with trap imperfections and special relativity, and variation in the magnetic field lead to statistical uncertainties in the average CFR that decrease as $N^{-1 / 2}$, where $N$ is the number of measurements. However, essentially all other effects discussed above, to the extent that they are not common to the two-ions, lead to systematic shifts in the CFR. For example, a systematic difference between the cyclotron radii of the two ions will result in a systematic shift to the CFR. Likewise, any differential equilibrium position shift and any imbalance in the image-charge, ion-detector interaction and ion-ion interaction shifts produce systematic shifts in the CFR. Some of these shifts can be characterized and corrected for, but usually the uncertainties in these corrections are not random and thus lead to systematic error. The range over which parameters such as the cyclotron radius can be varied and still allow good statistical precision is usually limited. This makes it difficult to accurately characterize some systematics.

\subsubsection{Mass Doublets and the Use of Molecular Ions: Stored Energy and Polarizability Shifts}

When measuring a cyclotron frequency ratio, with the exception of the dynamical part of the ion-ion interaction, all the above systematic errors tend to zero for two ions of the same charge as the difference in $m / q$ between the ions tends to zero. This motivates measurements between ions of the same total atomic number, which we call "mass-doublets". In most cases, to achieve this requires that one or both of the ions in the pair is a molecular ion. This leads to the complication that the molecular binding energy of the ions must be known [12], either by measurement or calculation.

If the ion is a homonuclear diatomic molecule, it can be in an excited vibrational state with a radiative lifetime of many days. For $\mathrm{H}_{2}{ }^{+}$the corresponding mass shifts are at the $\sim 5 \times 10^{-10}$ level [38,55], while for ions such as $\mathrm{N}_{2}{ }^{+}$and $\mathrm{O}_{2}{ }^{+}$they are $\sim 10^{-11}$. For higher precision, the metastable 
ro-vibrational levels must be identified. This has been done for the vibrational levels of $\mathrm{H}_{2}{ }^{+}$[55] and, partially, for highly excited rotational levels of $\mathrm{H}_{3}{ }^{+}$[56].

In the case of a non-homonuclear molecular ion, there can be a large body-frame electric dipole moment. This produces large rotational-state and sub-state dependent electric polarizability, particularly in the ground and the first-excited rotational states [52,57]. This polarizability leads to a shift in $f_{c}$ given approximately by [58]

$$
\Delta f_{c} / f_{c}=-9.93 \times 10^{-15} \alpha B^{2} / M,
$$

where, here, $\alpha$ is the polarizability in atomic units, $M$ is the mass of the ion in $\mathrm{u}$, and $B$ is the magnetic field in tesla. If the dipole moments are known from molecular structure calculations, the state-dependent polarizabilities can be calculated in the Born-Oppenheimer approximation [58]. Hence, if the rotational state of the ion can be identified, for example, if it is known that the ion is in the ground rotational level, or if a spectrum of "polarizability shifts" can be observed in the CFR data, corrections can be applied [15,57-61]. Allowance must also be made for black-body radiation induced transitions between rotational levels.

\section{Atomic Masses of Hydrogen and Helium Isotopes}

The atomic masses of the proton, deuteron, triton, helion, and alpha particle (the nuclei of the atoms $\mathrm{H}, \mathrm{D}, \mathrm{T},{ }^{3} \mathrm{He}$, and ${ }^{4} \mathrm{He}$ ) are considered to be fundamental constants and are included in the recent CODATA tabulations, (e.g., references [2,3]). The ratio of the electron mass to the masses of the proton and deuteron are needed for the analysis of precision laser spectroscopy of hydrogen and deuterium used to obtain the Rydberg constant. The fractional uncertainty of 1s-2s two-photon spectroscopy in hydrogen is currently $4 \times 10^{-15}$ [62]. In principle, to extract the Rydberg constant to this precision requires that $m_{e} / m_{p}$ be known to $<8 \times 10^{-12}$. However, at present, uncertainty in the proton charge radius dominates and the CODATA14 value for $R_{\infty}$ has an uncertainty of $5.9 \times 10^{-12}$ [2], relaxing the precision required for $m_{e} / m_{p}$ by three orders-of-magnitude. However, this situation could change if the so-called "proton radius puzzle" is resolved in such a way that confidence can be placed in the determination of the proton charge radius from the precise measurements of the Lamb-shift of muonic hydrogen [63].

An interesting development is the proposal to obtain $m_{e} / m_{p}$ and $m_{e} / m_{d}$ from precision spectroscopy of ro-vibrational transitions in $\mathrm{H}_{2}{ }^{+}$and $\mathrm{HD}^{+}$, in combination with precise theoretical calculations including relativistic and QED corrections, (e.g., references [64-66]). With further developments in the theory, this may lead to sub-10 11 values for the mass ratios, but also, by using combinations of transitions, to competitive values for $R_{\infty}$ and the charge radii of the proton and deuteron [67]. Additionally, with a sufficiently developed theory, measurements of the Zeeman-hyperfine structure and cyclotron frequency of $\mathrm{H}_{2}{ }^{+}$can be used to obtain the electron-to-proton mass ratio, and the magnetic moment of the proton [68]. All these projects motivate further measurements of $M[p]$ and $M[d]$ by Penning trap techniques.

The mass difference between tritium and helium- 3 is closely related to the $Q$-value (available energy) of the beta-decay of tritium. This is an important parameter for testing systematics in precision measurements of the beta-electron energy spectrum of tritium near its endpoint, which set upper limits on the mass of the electron neutrino [69,70]. For this reason, the masses of $\mathrm{T}^{+}$and ${ }^{3} \mathrm{He}^{+}$are often measured together so that some systematics cancel in their difference. A precision atomic mass of ${ }^{3} \mathrm{He}$ will also be needed to convert measurements of the ratio of the nuclear spin-flip frequency of ${ }^{3} \mathrm{He}^{2+}$ to its cyclotron frequency into a value for the nuclear $g$-factor of the helion [71]. The same will be true for the deuteron. A precise mass for ${ }^{4} \mathrm{He}$ is needed for measurements of the electronic $g$-factor of ${ }^{4} \mathrm{He}^{+}$, which can be used to obtain the electron mass and, possibly in the future, the fine-structure constant [72] (but also see Section 5). 
In what follows we show those mass measurements that have sufficient precision to influence the results of least-squares adjustments such as the AME or CODATA, sometimes with additional, less precise values for context. In general, we show the atomic masses as published in the original papers. In some cases, the results are based on CFRs relative ${ }^{12} \mathrm{C}$, while others use atoms other than ${ }^{12} \mathrm{C}$ as references. In the latter case, we attempt to show the effects of significant updates in the masses of the references. However, this is essentially illustrative and is not a substitute for a least-squares adjustment based on the relevant CFRs (or their equivalent mass difference equations) as is done by the AME.

\subsection{Proton}

Atomic mass results for the proton and the least-squares value from the AME2016 are shown in Table 1. The first of these, and the first with an uncertainty of $<10^{-9}$ was by the MIT group. Here the CFR of ${ }^{12} \mathrm{CH}_{4}{ }^{+}$versus ${ }^{12} \mathrm{C}^{+}$was measured using a two-cyclotron-pulse (Ramsey) variant of the PnP technique [73]. By mapping the final cyclotron phase onto cyclotron amplitude, both ions' cyclotron frequencies could be measured at the same trap voltage, thereby eliminating the equilibrium position shift error discussed in Section 2.3.3. The second is a result from the UW group [74], who compared a proton against $\mathrm{C}^{4+}$, using an exceptionally stable $6 \mathrm{~T}$ solenoid magnet and the swept cyclotron drive, axial-frequency-shift technique. Here, auxiliary measurements of $\mathrm{C}^{4+}$ against $\mathrm{C}^{5+}$ provided some check against the equilibrium-position and other systematic errors, and the error budget had contributions from anharmonicity (95 ppt), residual magnetic field drifts $(70 \mathrm{ppt})$, statistics (50 ppt), and image charge shifts (40 ppt). Also notable was the use of the high axial frequency of $3.5 \mathrm{MHz}$, which reduces some anharmonic shifts and leads to a lower cyclotron temperature after cyclotron-to-axial coupling.

Table 1. Atomic mass values for the proton.

\begin{tabular}{ll}
\hline Source & Atomic Mass (u) \\
\hline MIT 1993 [73] & $1.0072764664(7)$ \\
UW 1999 [74] & $1.00727646689(14)$ \\
SMILETRAP 2002 [75] & $1.00727646686(35)$ \\
SMILETRAP 2008 [38] & $1.00727646695(18)$ \\
MPIK* 2017 [46] & $1.007276466583(33)$ \\
AME2016 [5] & $1.007276466929(90)$ \\
\hline
\end{tabular}

The third is a measurement by the SMILETRAP group [75] using their time-of-flight detection technique to measure the CFRs of $\mathrm{H}_{2}{ }^{+}$relative to several charge states of ${ }^{12} \mathrm{C}$, but also of ${ }^{28} \mathrm{Si},{ }^{14} \mathrm{~N}$, ${ }^{20} \mathrm{Ne}$, and ${ }^{40} \mathrm{Ar}$, making use of measurements of these atoms at the $10^{-10}$ level by MIT [41]. The fourth is another measurement by the SMILETRAP group [38], but making use of the Ramsey variant of their TOF method, and measuring $\mathrm{H}_{2}{ }^{+}$against $\mathrm{D}^{+}$as a reference. To obtain the proton mass they assumed an average vibrational excitation energy of $0.74(7) \mathrm{eV}$ for $\mathrm{H}_{2}{ }^{+}$and a value for $M[d]=2.013553212$ 73(4) $\mathrm{u}$, as provided in a private communication from the UW group. (Using the updated mass for D from the UW group, see Table 2, increases this value for $M[p]$ by only $7.5 \mathrm{ppt}$.)

The fifth is a recent and advanced measurement with a fractional uncertainty of $3.3 \times 10^{-11}$ by the MPIK-Heidelberg/Mainz/GSI/RIKEN collaboration, using a purpose built, cylindrical, multi-Penning trap system for light ions, now called LIONTRAP [46]. Here the cyclotron frequencies of a proton and a ${ }^{12} \mathrm{C}^{6+}$ ion were measured, mainly using the PnA technique, but with checks by the double-dip technique, and by alternately shuttling the two ions into a "measurement" trap from reservoir traps on either side. A quadratic potential could be produced over a relatively large volume in the measurement trap by incorporating two sets of compensation electrodes. The equilibrium position shift was avoided by using two axial detectors, one resonant at about $525 \mathrm{kHz}$ for ${ }^{12} \mathrm{C}^{6+}$ and the other at $740 \mathrm{kHz}$ for the proton, so the measurements of $f_{c}{ }^{\prime}, f_{z}$ and $f_{m}$ could be carried out at the same trap voltage. By using the PnA technique, and reducing the effective temperature of the axial motion using feedback cooling, a proton cyclotron radius as small as $9 \mu \mathrm{m}$ could be used. Hence relativistic shifts to the 
cyclotron frequency were reduced and the estimated systematic uncertainty due to special relativity on the CFR was 7 ppt. Overall, the largest uncertainty was from the finite axial motion, mainly of the proton, combined with the quadratic magnetic field inhomogeneity $B_{2}$, which gave $27 \mathrm{ppt}$. The model of systematics was also checked by comparing ${ }^{12} \mathrm{C}^{3+}$ to ${ }^{12} \mathrm{C}^{6+}$ and the proton to ${ }^{16} \mathrm{O}^{8+}$. As Table 1 shows, the MPIK* result is in a 2 standard-deviation disagreement with both the UW and the second SMILETRAP result and indicates a lighter proton. However, the SMILETRAP-MPIK* disagreement might also indicate that the deuteron is lighter than as measured by the UW group, see next section.

New measurements of $\mathrm{H}_{2}{ }^{+} / \mathrm{D}^{+}$using two simultaneously trapped ions are currently in progress at FSU [55]. Unlike the SMILETRAP TOF technique in which the measurements were carried out on $\mathrm{H}_{2}{ }^{+}$within a few seconds after leaving the ion source, the $\mathrm{H}_{2}{ }^{+}$is trapped for many days and can be Stark-quenched to the vibrational ground state [76]. This removes the uncertainty due to the vibrational distribution of the $\mathrm{H}_{2}{ }^{+}$that had to be assumed in [38].

Table 2. Atomic mass values for the deuteron.

\begin{tabular}{|c|c|}
\hline Source & Atomic Mass (u) \\
\hline MIT 1993 [73] & $2.0135532126(6)$ \\
\hline UW 2015 [77] & $2.013553212745(40)$ \\
\hline SMILETRAP $2008[38,46]^{1}$ & $2.01355321200(36)$ \\
\hline AME2016 [5] & $2.01355321280(12)$ \\
\hline
\end{tabular}

${ }^{1}$ Using the MPIK* result of Table 1 for $M[p]$ [46] as the reference.

\subsection{Deuteron and Neutron}

\subsubsection{Deuteron}

Table 2 shows the results for the mass of the deuteron. Similar to the proton mass, the MIT measurement [73] determined the ratio of ${ }^{12} \mathrm{CD}^{4+}$ to ${ }^{12} \mathrm{C}+$ using the Ramsey technique at constant trap voltage. The more precise UW result, with fractional uncertainty of $2 \times 10^{-11}$ [77], is from the final analysis of data taken a decade earlier using similar techniques as for their proton measurement. A significant advantage compared to the proton is that $\mathrm{D}^{+}$was measured against ${ }^{12} \mathrm{C}^{6+}$, which has the same nominal $\mathrm{m} / \mathrm{q}$. The analysis used data from 10 runs, each taking about a month including tests for systematics. The error budget had comparable contributions from uncertainty in the axial amplitude combined with trap imperfections, the fits to the axial-frequency error signal versus drive frequency, the phase of the phase-locked-loop that is used to detect the shifts in axial frequency, and from image charge. The image charge shift was estimated at $245 \mathrm{ppt}$, but its uncertainty was estimated to be $10 \mathrm{ppt}$ in the CFR. Only the uncertainty in the image charge was treated as a systematic error (that is, not reduced in the average). In the third row, we show the result of combining the $\mathrm{D}^{+} / \mathrm{H}_{2}{ }^{+}$ ratio as measured by SMILETRAP (the fourth result for the proton in Table 1) [38] with the MPIK* 2017 proton mass [46], to give a mass for the deuteron. As can be seen, this results in a deuteron mass 0.75(36) nu smaller than the UW value.

\subsubsection{Neutron}

The mass of the neutron is obtained from the wavelength of the $2.2 \mathrm{MeV}$ gamma ray emitted in the (non-resonant) neutron capture reaction $n+p \rightarrow d+\gamma$, combined with the masses of $p$ and $d$. Using the Bragg-crystal diffraction wavelength result obtained by a National Institute of Standards and Technology (NIST) and Institut Laue-Langevin (ILL) collaboration [78], subsequently corrected following a re-evaluation of the crystal lattice spacing, and then for the conversion between wavelength and $\mathrm{u}$, Huang et al. obtained an equivalent mass difference $p+n-d=2388$ 169.95(42) nu [4]. The result of combining this deuteron binding energy with the UW mass for $d$ [77] and the 2017 MPIK* mass for $p$ [46] is compared with the AME2016 result in Table 3. As can be seen, the reduced proton mass 
results in a neutron mass increased by $\sim 0.3 \mathrm{nu}$, but the change is less than the uncertainty due to the wavelength measurement.

Table 3. Atomic mass values for the neutron.

\begin{tabular}{ll}
\hline Source & Atomic Mass (u) \\
\hline NIST/ILL + UW + MPIK* (see text) & $1.00866491611(42)$ \\
AME2016 [5] & 1.008664915 80(50) \\
\hline
\end{tabular}

\subsection{Tritium and Helium-3}

In Table 4 we show values for the atomic masses of tritium and helium-3 as given in the respective publications. (The atomic mass of the triton can be obtained from the mass of the tritium atom by subtracting $5.48565305257(16) \times 10^{-4} \mathrm{u}$, and that of the helion from helium-3 by subtracting $\left.1.097075006640(32) \times 10^{-3} \mathrm{u}\right)$.

Table 4. Atomic masses of tritium and helium-3 as originally reported.

\begin{tabular}{lll}
\hline Source & Tritium $(\mathbf{u})$ & Helium-3 (u) \\
\hline SMILETRAP 2006 [79] & $3.0160492787(25)$ & $3.0160293217(26)$ \\
FSU 2015 [32] & $3.01604928178(19)$ & $3.01602932243(19)$ \\
UW 2015 [77] & & $3.016029321675(43)$ \\
\hline
\end{tabular}

The first row gives the results of the SMILETRAP group from CFRs of ${ }^{3} \mathrm{He}^{+},{ }^{3} \mathrm{He}^{2+}$, and $\mathrm{T}^{+}$ measured against $\mathrm{H}_{2}{ }^{+}$using the TOF technique [79]. Here the $\mathrm{H}_{2}{ }^{+}$ions, allowing for vibrational excitation energy, were assumed to have an average mass of 2.015101497 03(27) $u$ as given in reference [34].

The second row gives the results from the FSU group of CFR measurements of ${ }^{3} \mathrm{He}^{+}$and $\mathrm{T}^{+}$against $\mathrm{HD}^{+}$[32]. For both $\mathrm{HD}^{+} /{ }^{3} \mathrm{He}^{+}$and $\mathrm{HD}^{+} / \mathrm{T}^{+}$, both ions were simultaneously trapped, but alternated between large and small cyclotron radii, the cyclotron frequency measurement being carried out on the inner ion using the PnP technique. The results were obtained from 34 measurements of $\mathrm{HD}^{+} /{ }^{3} \mathrm{He}^{+}$ and 31 of $\mathrm{HD}^{+} / \mathrm{T}^{+}$, each lasting 8 to $10 \mathrm{~h}$, resulting in a statistical uncertainty of $17 \mathrm{ppt}$ for each ratio. The $\mathrm{HD}^{+}$mass was calculated using the CODATA2010 masses for the proton and deuteron [80], and the very precise non-relativistic $\mathrm{HD}^{+}$binding energy given in reference [81]. In addition, the CFR was corrected by $9.4 \times 10^{-11}$ to allow for the polarizability of the $\mathrm{HD}^{+}$molecular ion $[58,81]$, with the assumption it remains in its ground rotational and vibrational level. Because the two CFRs differ from 1 by only $0.2 \%$, most systematic errors were estimated to be negligible. However, a surprisingly large $3 \%$ imbalance was observed in the cyclotron radii, which was assumed to be due to a strong frequency dependence in the cyclotron drive transfer function. This led to a common systematic uncertainty of 45 ppt being assigned to both ratios.

The third row gives the result of a measurement of ${ }^{3} \mathrm{He}^{2+}$ against ${ }^{12} \mathrm{C}^{6+}$ and ${ }^{12} \mathrm{C}^{5+}$ by the UW group, which was reported in the same publication as the measurements on $\mathrm{D}^{+}$[77]. The data consisted of 4 runs of ${ }^{3} \mathrm{He}^{2+}$ versus ${ }^{12} \mathrm{C}^{6+}$, of which two involved an extra contaminant ${ }^{12} \mathrm{C}^{6+}$ ion, and 3 of ${ }^{3} \mathrm{He}^{2+}$ versus ${ }^{12} \mathrm{C}^{5+}$, all of which are non-doublets. The error budget contains similar contributions as for the measurements on the deuteron, with a common systematic uncertainty of 9 ppt on an estimated image charge shift of 220 ppt.

Since the SMILETRAP publication [79] and the FSU publication [32], improved values for the mass of the proton and deuteron have been published. In the case of the SMILETRAP result, it is possible to bypass uncertainty due to the mass of $\mathrm{H}_{2}{ }^{+}$, both due to the proton mass and the average excitation energy, by using their $\mathrm{H}_{2}{ }^{+} / \mathrm{D}^{+}$measurement in reference [38] to relate $\mathrm{T}^{+}$and ${ }^{3} \mathrm{He}^{+}$to $\mathrm{D}^{+}$. Hence, the latest UW result for $\mathrm{D}^{+}$[77] can be used as the reference. Making these adjustments, the corrected $\mathrm{T}$ and ${ }^{3} \mathrm{He}$ masses are shown in the first row of Table 5. Further, and significantly, there has 
been a re-measurement of $\mathrm{HD}^{+} /{ }^{3} \mathrm{He}^{+}$by the FSU group using a substantially rebuilt apparatus, with confirmation by taking the ratio of the two CFRs ${ }^{3} \mathrm{He}^{+} / \mathrm{H}_{3}{ }^{+}$and $\mathrm{HD}^{+} / \mathrm{H}_{3}{ }^{+}$[61]. The result is in good agreement with the 2015 result [32], but a factor of two more precise. Because systematic errors in both the 2017 and 2015 measurements are likely to be common, the new $\mathrm{HD}^{+} /{ }^{3} \mathrm{He}^{+}$ratio supersedes the previous one. However, the 2015 result can still be used to relate the mass of $\mathrm{T}$ to the new mass of ${ }^{3} \mathrm{He}$. Hence, using the new $\mathrm{HD}^{+} /{ }^{3} \mathrm{He}^{+}$ratio from reference [61], the $\mathrm{T}^{+} /{ }^{3} \mathrm{He}^{+}$ratio from reference [32], the deuteron mass from reference [77], and the proton mass from reference [46], we obtain the results given in the third row of Table 5. For ease of comparison, in the second row, we have copied the UW result for ${ }^{3} \mathrm{He}$ [77] from Table 4.

Table 5. Updated atomic masses of tritium and helium-3 (see text).

\begin{tabular}{lll}
\hline Source & Tritium $(\mathbf{u})$ & Helium-3 (u) \\
\hline SMILETRAP [38,79] & $3.0160492789(25)$ & $3.0160293219(26)$ \\
UW 2015 [77] & & $3.016029321675(43)$ \\
FSU 2017 [32,61] & $3.01604928148(11)$ & $3.01602932214(9)$ \\
AME 2016 [5] & $3.01604928199(23)$ & $3.01602932265(22)$ \\
\hline
\end{tabular}

As can be seen from Tables 4 and 5, the FSU mass of ${ }^{3} \mathrm{He}$, while being consistent with the lower precision SMILETRAP result, is significantly higher than the UW result. And, although allowing for the updated proton mass has reduced the discrepancy from $0.76(0.19)$ nu to $0.47(0.1) \mathrm{nu}$, because of the increased precision, the discrepancy is still 4 standard deviations. Additional evidence for a heavier ${ }^{3} \mathrm{He}$ than that reported in reference [77] is provided by referring the mass of ${ }^{3} \mathrm{He}$ to that of the proton only, using the $\mathrm{H}_{3}{ }^{+}$ion [56]. In this case, because the $\mathrm{H}_{3}{ }^{+}$may have significant stored rotational energy, only an upper limit to $3 M[p]-M[h]$ can be given. However, if this is done, using the proton mass of reference [46] as a reference, the result is $M\left[{ }^{3} \mathrm{He}\right]>3.01602932200(14) \mathrm{u}$, which is inconsistent with the UW result [77] by (more than) $3.3(1.5) \times 10^{-10} \mathrm{u}$. We note that the discrepancy would be nearly removed if the FSU CFRs involving $\mathrm{HD}^{+}$were not corrected for the polarizability of the $\mathrm{HD}^{+}$ ion, which would be appropriate if the $\mathrm{HD}^{+}$did not spend the majority of time in its ground-state during the measurement. However, this is inconsistent with the fact that the first excited rotational level of $\mathrm{HD}^{+}$, which has a mean lifetime of $150 \mathrm{~s}$, has an excitation energy corresponding to $63 \mathrm{~K}$, while the trap electrodes are at $4.2 \mathrm{~K}$. The AME2016 results are $0.5 \mathrm{nu}$ higher than the updated FSU results because they were based on the 2015 FSU results and lacked the updated proton mass of reference [46].

In Table 6, we show the mass differences between atoms of tritium and helium-3 expressed in $\mathrm{eV}$. It is important to note that several important systematic errors cancel for this difference, so the uncertainty is smaller than that obtained from the uncertainties in the individual masses. The first row gives this difference as measured by the UW group [82], using an earlier apparatus than that used for the other measurements discussed in this review. (The absolute masses for ${ }^{3} \mathrm{He}$ and $\mathrm{T}$ reported in reference [82] were found to be in error by several standard deviations by the SMILETRAP group, but the relevant systematic error at least partly cancels for the $\mathrm{T}-{ }^{3} \mathrm{He}$ difference.) The second row gives the result from the 2006 SMILETRAP measurements [79], and the third gives the more than a factor of 10 more precise result from the FSU group [32]. This result is higher than the SMILETRAP result by 2.2(1.2) eV and its uncertainty is slightly less than that of the absolute calibration of the retarding voltage in KATRIN, which is now $90 \mathrm{meV}$ [70].

Table 6. Mass difference between atoms of tritium and helium-3, expressed in eV.

\begin{tabular}{ll}
\hline Source & $\left.M[T]-M{ }^{3} \mathbf{H e}\right] \mathbf{e V}$ \\
\hline UW 1993 [82] & $18590.1(1.7)$ \\
SMILETRAP 2006 [79] & $18589.8(1.2)$ \\
FSU 2015 [32] & $18592.01(0.07)$ \\
\hline
\end{tabular}




\subsection{Helium-4}

The three most recent determinations of the atomic mass of ${ }^{4} \mathrm{He}$ are shown in Table 7 . (The mass of the alpha-particle can be obtained by subtracting $1.097075002640(32) \times 10^{-3} \mathrm{u}$ ). The first two, from Mainz and SMILETRAP, respectively, used the TOF detection technique with $\mathrm{H}_{2}{ }^{+}$and $\mathrm{D}_{2}{ }^{+}$as references. The third is a much more precise $\left(1.6 \times 10^{-11}\right.$ fractional precision) measurement of the CFR of ${ }^{4} \mathrm{He}^{2+}$ against ${ }^{12} \mathrm{C}^{6+}$ (and so a mass doublet) by the UW group [83], using their swept-cyclotron-drive axial-frequency-shift detection technique. The estimated systematic uncertainty was $9 \mathrm{ppt}$ from image charges and $13.4 \mathrm{ppt}$ from all other sources. The two TOF measurements disagree with each other by 7.9(2.6) nu, and the more precise TOF measurement disagrees with the cryogenic Penning trap result by $2.7(1.3) \mathrm{nu}$.

Table 7. Atomic mass values for ${ }^{4} \mathrm{He}$.

\begin{tabular}{ll}
\hline Source & Atomic Mass (u) \\
\hline Mainz 2001 [84] & $4.0026032489(22)$ \\
SMILETRAP 2001 [85] & $4.0026032568(13)$ \\
UW 2004 [83] & $4.002603254153(64)$ \\
AME 2016 [5] & $4.00260325413(6)$ \\
\hline
\end{tabular}

\section{Atomic Masses of ${ }^{87} \mathrm{Rb}$ and ${ }^{133} \mathrm{Cs}$ for the Atom-Recoil Method for the Fine-Structure Constant}

The fine-structure constant $\alpha\left(=e^{2} /\left(2 \varepsilon_{0} h c\right)\right.$ is the dimensionless constant that characterizes the strength of the electromagnetic interaction. In the new-SI, with $e$ and $h$ fixed, its measurement determines the permittivity of free space $\varepsilon_{0}$, which is no longer fixed. Currently, the two most precise methods for obtaining $\alpha$ are based on measurements of the anomaly of the $g$-factor of the free electron, i.e., its difference from the Dirac value of 2; and, the recoil velocity of atoms having absorbed a photon of light. In the former case, $\alpha$ is obtained by combining a complex Quantum Electrodynamics (QED) calculation of $g_{e}-2$ in terms of $\alpha$ up to 10-th order [86], with a precise measurement using a single electron in a cryogenic Penning trap [87].

Regarding the second method, the atom-recoil measurements yield values for $h / m_{\text {atom }}$, where $m_{\text {atom }}$ is the absolute mass of the particular atom. The most precise measurements have been performed with ${ }^{87} \mathrm{Rb}$ [88] and ${ }^{133} \mathrm{Cs}$ [89] using atom-interferometric techniques. These resulted in values for $h / m_{\text {atom }}$ with fractional uncertainties of $1.2 \times 10^{-9}$ and $4 \times 10^{-10}$, respectively. From these measurements, $\alpha$ is obtained using the relation (c.f. Equation (1) above)

$$
\alpha^{2}=\left(2 R_{\infty} / c\right)\left(h / m_{\text {atom }}\right)(M[a t o m] / M[e])
$$

Hence, to obtain $\alpha$, in addition to the Rydberg constant, measured at $6 \times 10^{-12}$ relative precision [2], and the atomic mass of the electron, measured to $2.9 \times 10^{-11}$ (see the next section), it is also necessary to know the atomic mass of the particular atom.

Table 8 shows the three most precise values for the fine-structure constant as obtained from $g_{e}-2,{ }^{87} \mathrm{Rb}$, and ${ }^{133} \mathrm{Cs}$, respectively. The ${ }^{87} \mathrm{Rb}$ result [88] has been updated using the latest value for $M[e]$ (the fourth entry in Table 10), and the AME2016 [2] value for $M\left[{ }^{87} \mathrm{Rb}\right]$. As can be seen, while the two atom-recoil results agree, there is a 2.4 sigma tension between the $g_{e}-2$ result and the ${ }^{133} \mathrm{Cs}$ result. As noted in reference [89], this level of (dis)-agreement provides information on physics beyond the Standard model and thus strongly motivates further related measurements.

The most recent measurements of the atomic masses of ${ }^{87} \mathrm{Rb}$ and ${ }^{133} \mathrm{Cs}$ are shown in Table 9. The SMILETRAP result [90] made use of high-charge states of ${ }^{133} \mathrm{Cs}(36+$ to $42+$ ) measured against ${ }^{12} \mathrm{C}^{4+}$ and $\mathrm{H}_{2}{ }^{+}$using their TOF technique. The uncertainty in the binding energy corrections for these highly-charged ions was estimated to be $\sim 10 \mathrm{eV}$, corresponding to $\sim 10^{-10}$ of the Cs mass. The result was presented as a mass ratio with respect to the proton and has been converted here into $M\left[{ }^{133} \mathrm{Cs}\right]$ using the new proton mass of reference [46]. Both the MIT [91] and the FSU results [31] used the 
PnP method in the same Penning trap, but with several differences of technique. In particular, MIT, using the make-and-remake technique mentioned in Section 2.3.1, measured the CFRs ${ }^{87} \mathrm{Rb}^{2+} / \mathrm{C}_{3} \mathrm{H}_{7}{ }^{+}$, ${ }^{87} \mathrm{Rb}^{2+} / \mathrm{C}_{3} \mathrm{H}_{8}{ }^{+},{ }^{133} \mathrm{Cs}^{2+} / \mathrm{C}_{5} \mathrm{H}_{6}{ }^{+}$, and ${ }^{133} \mathrm{Cs}^{3+} / \mathrm{CO}_{2}{ }^{+}$. For reasons that were not understood, run-to-run scatter exceeded the estimated statistical error based on the data fit for each run. One might also be concerned about polarizability shifts for the hydrocarbon molecular reference ions. However, when averaged over all rotational sub-states, the only remaining contribution to polarizability from the body-frame dipole moment (i.e., "orientation polarizability") is from the ground-state [58], which has a small probability of occupation for these ions.

Table 8. Recent results for the inverse fine-structure constant.

\begin{tabular}{ll}
\hline Source & $\mathbf{1 / \alpha}$ \\
\hline$g_{e}-2$ 2018 [86,87] & $137.035999149(33)$ \\
${ }^{87} \mathrm{Rb} 2011[88]^{1}$ & $137.035998995(85)$ \\
${ }^{133} \mathrm{Cs} 2018[89]$ & $137.035999046(27)$ \\
CODATA 2014 [2] & $137.035999139(31)$ \\
\hline \multicolumn{2}{c}{${ }^{1}$ With updated value for $M[e]}$.
\end{tabular}

Table 9. Atomic mass values for ${ }^{87} \mathrm{Rb}$ and ${ }^{133} \mathrm{Cs}$.

\begin{tabular}{lll}
\hline Source & Rubidium-87 & Cesium-133 \\
\hline SMILETRAP 1999 [90] & & $132.90545188(28)$ \\
MIT 1999 [91] & $86.909180520(15)$ & $132.905451931(27)$ \\
FSU 2010 [31] & $86.909180535(10)$ & $132.905451963(13)$ \\
AME 2016 [5] & $86.909180531(6)$ & $132.905451961(9)$ \\
\hline
\end{tabular}

In the case of the FSU results [30], the masses of ${ }^{87} \mathrm{Rb}$ and ${ }^{133} \mathrm{Cs}$ were related, robustly, to those of ${ }^{86} \mathrm{Kr}$ and ${ }^{129,132} \mathrm{Xe}$ by measuring ${ }^{87} \mathrm{Rb}^{2+} /{ }^{86} \mathrm{Kr}^{2+},{ }^{133} \mathrm{Cs}^{3+} /{ }^{132} \mathrm{Xe}^{3+}$ and ${ }^{133} \mathrm{Cs}^{3+} /{ }^{129} \mathrm{Xe}^{3+}$ using the technique of two simultaneously trapped ions, and alternating between large and small cyclotron orbits. The masses of ${ }^{86} \mathrm{Kr}$ and ${ }^{129,132} \mathrm{Xe}$ had previously been obtained from extensive measurements using the make-and-remake technique of ${ }^{84,86} \mathrm{Kr}$ and ${ }^{129,132} \mathrm{Xe}$, in several charge states, against ${ }^{12} \mathrm{C}^{16} \mathrm{O}_{2}{ }^{+}$, ${ }^{12} \mathrm{C}_{2} \mathrm{H}_{2}{ }^{+}$and ${ }^{14} \mathrm{~N}_{2}{ }^{+}$[30]. Here, the mass of ${ }^{16} \mathrm{O}$ was taken from the AME2003 value [92], which was mostly based on the UW result [40] (see Table 11), while that of ${ }^{14} \mathrm{~N}$ was also referenced to ${ }^{16} \mathrm{O}$, using the ultra-precise MIT simultaneous measurement result for ${ }^{14} \mathrm{~N}_{2}{ }^{+} / \mathrm{CO}^{+}$[57]. The FSU and MIT results are in good agreement, and the fractional uncertainties of the FSU results, $1.2 \times 10^{-10}$ and $1.0 \times 10^{-10}$, are a modest improvement for ${ }^{87} \mathrm{Rb}$ and a factor of 2 improvement for ${ }^{133} \mathrm{Cs}$, respectively. We note that if one were to explain the above 2.4 sigma discrepancy between the $g_{e}-2$ and ${ }^{133} \mathrm{Cs}$ values for $\alpha$ as due to an error in the ${ }^{133} \mathrm{Cs}$ mass, this error would need to be $\sim 1.5 \times 10^{-9}$. Atom-interferometric techniques for measuring $h / m_{\text {atom }}$ are being developed for various isotopes of other atoms including $\mathrm{Li}$ [93], Sr [94], and $\mathrm{Yb}$ [95], motivating measurements of the relevant atomic masses [31,96].

\section{Atomic Mass of the Electron from the $g$-Factor of Hydrogen-Like Ions and Future Possibilities for Measuring the Fine-Structure Constant}

\subsection{Atomic Mass of the Electron}

The atomic mass of the electron has been obtained by the UW group, who compared the cyclotron frequencies of small clouds (5 to 13) of electrons and a ${ }^{12} \mathrm{C}^{6+}$ ion in a Penning trap [97], see the first row of Table 10. 
Table 10. Atomic mass values for the electron.

\begin{tabular}{ll}
\hline Source & Atomic Mass (u) \\
\hline UW 1995 [97] & $0.0005485799111(12)$ \\
Mainz 2002 ${ }^{12} \mathrm{C}^{5+}[98]$ & $0.0005485799092(4)$ \\
Mainz 2004 ${ }^{16} \mathrm{O}^{7+}[99]$ & $0.0005485799096(4)$ \\
MPIK* 2014 ${ }^{12} \mathrm{C}^{5+}$ & $0.000548579909065(16)$ \\
[100,101] & $0.000548579909070(16)$ \\
\hline
\end{tabular}

Here, the precision was limited by the line shape in the swept-cyclotron drive method and by the large relativistic shift to the cyclotron frequency of the electron due to its small mass. Using quantum-jump techniques with a single electron in a trap cooled by a dilution refrigerator, as applied to the latest measurements of $g_{e}-2$ [87], a greatly improved measurement of the CFR of an electron relative to a negative ion such as $\mathrm{H}^{-}$or $\mathrm{C}^{-}$should be possible.

However, as initially developed at Mainz, the uncertainty due to SR has instead been overcome by extracting the electron mass from precise measurements of the $g$-factor of the electron bound in hydrogen-like ions such as ${ }^{12} \mathrm{C}^{5+}$ and ${ }^{16} \mathrm{O}^{7+}$ [98-100] combined with bound-state QED theory, (e.g., references [101,102]). In the measurements, which have been recently reviewed in this journal [103], the microwave frequency that flips the ion's electron spin in a magnetic field is determined at the same time as the ion's cyclotron frequency. Since the electron spin-flip frequency $f_{s f}$ can be written in terms of the electronic $g$-factor of the hydrogen-like ion $g_{i o n}$ as $f_{s f}=\left(g_{\text {ion }} \mu_{\mathrm{B}} / h\right) B$, where $\mu_{\mathrm{B}}=e h /\left(4 \pi m_{e}\right)$ is the Bohr magneton, and the cyclotron frequency is given by $f_{c}=(1 / 2 \pi)\left(q_{\text {ion }} / m_{\text {ion }}\right) B$, it follows that

$$
m_{e} / m_{\text {ion }}=\left(g_{\text {ion }} / 2\right)\left(e / q_{\text {ion }}\right)\left(f_{c} / f_{s f}\right) .
$$

Hence, if $g_{\text {ion }}$ can be accurately calculated, as is the case for lower- $Z$ hydrogen-like ions [101], $m_{e} / m_{i o n}$ can be determined from a measurement of $f_{s f} / f_{c}$. If the ion is ${ }^{12} \mathrm{C}^{5+}$, after allowance for ionization energies, this gives the atomic mass of the electron. Otherwise, the atomic mass of the atom must be measured separately. Results of early experiments at Mainz on $\mathrm{C}^{5+}$ and $\mathrm{O}^{7+}$ are shown in the second and third rows of Table 10. These values change slightly if the updated theory for the $\mathrm{g}$-factor is used [101]. Using refinements such as the PnA method to measure the cyclotron frequency, a second and considerably more precise measurement of the $g$-factor of $\mathrm{C}^{5+}$ has been carried out by Sturm et al. [100]. Here, the largest contribution to uncertainty was $25 \mathrm{ppt}$ from statistics, and the dominant systematic error was the 14 ppt uncertainty in the image charge correction of $282 \mathrm{ppt}$. In future work, this could be reduced by using a larger Penning trap. When combined with the updated theory for the $g$-factor [101], this gives the result for the electron mass at $3 \times 10^{-11}$ relative precision shown in the last row of Table 10. This result is a fraction 3.6(2.0) $\times 10^{-9}$ lower than the UW result based on the CFR measurement.

\subsection{Atomic Masses for $\mathrm{g}$-Factor Measurements for the Electron Mass}

Equation (5) applied to ions other than ${ }^{12} \mathrm{C}^{5+}$ requires precise measurements of the atomic masses of the ions. In addition to the measurement on ${ }^{16} \mathrm{O}^{7+}$ for $M[e]$ [99], a measurement of $f_{s f} / f_{c}$ on ${ }^{28} \mathrm{Si}^{13+}$ [104] with a fractional uncertainty of $5 \times 10^{-11}$ was carried out to provide information on the difficult to calculate, higher-order terms in in the QED theory of the $g$-factor that scale with high powers of Z. In Table 11, we show some of the recent measurements of the atomic masses of ${ }^{16} \mathrm{O}$ and ${ }^{28} \mathrm{Si}$. $\left({ }^{16} \mathrm{O}\right.$ is also important as a reference for FSU and MIT measurements of ${ }^{87} \mathrm{Rb}$ and ${ }^{133} \mathrm{Cs}$ for the fine-structure constant, see Section 4.) 
Table 11. Atomic mass values for ${ }^{16} \mathrm{O}$ and ${ }^{28} \mathrm{Si}$.

\begin{tabular}{lll}
\hline Source & Oxygen-16 (u) & Silicon-28 (u) \\
\hline MIT 1995 [41] & $15.9949146195(21)$ & $27.9769265324(20)$ \\
UW [40] & $15.99491461946(16)$ & \\
FSU [15] & & $27.9769265350(6)$ \\
AME2016 [5] & $15.99491461960(17)$ & $27.9769265350(5)$ \\
\hline
\end{tabular}

As can be seen, the MIT and UW results for ${ }^{16} \mathrm{O}$ are in good agreement and both are adequately precise for the ${ }^{16} \mathrm{O}^{7+} g$-factor measurement [99]. The MIT result [41] was obtained from a set of interlocking ratios using the make-and-remake technique, of which ${ }^{12} \mathrm{CH}_{4}{ }^{+} /{ }^{16} \mathrm{O}^{+},{ }^{12} \mathrm{C}_{2} \mathrm{H}_{4}{ }^{+} /{ }^{12} \mathrm{C}^{16} \mathrm{O}^{+}$, and $\mathrm{C}_{3} \mathrm{H}_{8}{ }^{+} / \mathrm{CO}_{2}{ }^{+}$had the most influence [21]. The UW result, at $1.0 \times 10^{-11}$ fractional precision, involved comparisons of ${ }^{16} \mathrm{O}^{6+}$ against ${ }^{12} \mathrm{C}^{4+}$ and ${ }^{12} \mathrm{C}^{6+}$ using the swept cyclotron frequency scheme. For ${ }^{28} \mathrm{Si}$, the FSU and MIT results, both using the PnP technique, are in reasonable agreement. The MIT result was based on the doublet CFRs ${ }^{12} \mathrm{C}_{2} \mathrm{D}_{2} \mathrm{H}_{2}{ }^{+} /{ }^{28} \mathrm{SiH}_{2}{ }^{+}$and ${ }^{15} \mathrm{~N}_{2}{ }^{+} /{ }^{28} \mathrm{SiH}_{2}{ }^{+}$(with ${ }^{12} \mathrm{CH}_{3}{ }^{+} /{ }^{15} \mathrm{~N}^{+}$ and $\left.{ }^{12} \mathrm{C}_{2} \mathrm{D}_{2} \mathrm{H}_{2}{ }^{+} /{ }^{15} \mathrm{~N}_{2}{ }^{+}\right)$. The more precise $\left(2.2 \times 10^{-11}\right.$ fractional precision) FSU measurement made use of simultaneously trapped ions, alternating between large and small cyclotron radii, and obtained $M\left[{ }^{28} \mathrm{Si}\right]$ from the doublet CFRs ${ }^{12} \mathrm{C}_{2} \mathrm{H}_{4}{ }^{+} /{ }^{28} \mathrm{Si}^{+},{ }^{13} \mathrm{C}_{2}{ }^{+} \mathrm{H}_{2}{ }^{+} /{ }^{28} \mathrm{Si}^{+},{ }^{28} \mathrm{SiH}_{3}{ }^{+} /{ }^{31} \mathrm{P}^{+}$, and ${ }^{16} \mathrm{O}_{2}{ }^{+} /{ }^{31} \mathrm{PH}^{+}$[15]. Hence, the FSU ${ }^{28}$ Si result has sensitivity to the UW result for ${ }^{16} \mathrm{O}$, both directly, and also through the ultra-precise MIT results for ${ }^{13} \mathrm{C}_{2} \mathrm{H}_{2}{ }^{+} /{ }^{14} \mathrm{~N}_{2}{ }^{+}$[43] and ${ }^{12} \mathrm{C}^{16} \mathrm{O}^{+} /{ }^{14} \mathrm{~N}_{2}{ }^{+}$[57], which link ${ }^{13} \mathrm{C}$ to ${ }^{16} \mathrm{O}$.

We also note that the theory for the $g$-factor of ${ }^{4} \mathrm{He}^{+}$, due to its low $\mathrm{Z}$, has smaller theoretical uncertainties and also smaller uncertainties due to nuclear size than ${ }^{12} \mathrm{C}^{5+}$. This motivates measurements of $f_{s f} / f_{c}$ and additional measurements of $M\left[{ }^{4} \mathrm{He}\right]$.

\subsection{Obtaining the Fine-Structure Constant from g-Factors of Highly-Charged Ions}

It has been proposed by several authors, (e.g., see reference [105]), that since the theoretical expression for $g_{i o n}$ in Equation (5) is a function of $\alpha$ and $Z \alpha$, measurements of the $g$-factors of hydrogen-like ions of different $Z$ can be combined to provide values for $\alpha$ as well as $M[e]$, provided the theoretical difficulties can be overcome. Fortuitously, due to cancellations in the theory, the $g$-factor for ${ }^{12} \mathrm{C}^{5+}$ is only weakly dependent on $\alpha$, and the current uncertainty in $\alpha$ contributes to the theoretical uncertainty in the $g$-factor at only the $\sim 10^{-13}$ level, see Figure 5 of reference [72] (but note that the uncertainty in the current value of $\alpha$ is a factor of 14 times smaller than that used in reference [72]). At a higher $Z$, there is a much greater sensitivity to $\alpha$, but also to uncertainty in the nuclear charge distribution. One strategy to overcome this is to make measurements on additional charge-states, such as lithium-like and boron-like, which enables much of the uncertainty due to nuclear size to be cancelled. Here too, precise atomic masses will be required. It was also noted in reference [72] that the nuclear size correction for the $g$-factor of ${ }^{4} \mathrm{He}^{+}$, uniquely, results in a smaller uncertainty in $g$, about $10^{-13}$, than that due to $\alpha$, which is currently $\sim 5 \times 10^{-13}$. So, at least in principle, a measurement of $f_{s f} / f_{c}$ on ${ }^{4} \mathrm{He}^{+}$at the (extreme) $10^{-13}$ level could produce a competitive value for $\alpha$, provided there is an equally precise value for $M[e] / M\left[{ }^{4} \mathrm{He}\right]$.

\section{Atomic Mass of Si for the XRCD Technique and of He and Ar for the Boltzmann Constant}

The X-ray-crystal-density (XRCD) method mentioned in the introduction, that formerly measured $N_{A}$ and which can now be used to realize the $\mathrm{kg}$, requires the atomic mass of ${ }^{28} \mathrm{Si}$, and also of ${ }^{29,30} \mathrm{Si}$ if an isotopically impure sample is used. Acoustic gas thermometry (AGT) [2], which formerly measured the Boltzmann constant $k_{B}$ and which now realizes the kelvin, generally uses either ${ }^{4} \mathrm{He}$ or ${ }^{40} \mathrm{Ar}$, which may have fractions of ${ }^{36,38} \mathrm{Ar}$, requiring atomic masses for these too. However, since all these masses have uncertainties in the AME 2016 less than $10^{-9}$, except ${ }^{38} \mathrm{Ar}$ which has $5.5 \times 10^{-9}$, and the precision of the XRCD technique is at the $10^{-8}$ level, and of AGT is at the $10^{-6}$ level, the uncertainties in these atomic masses are not an issue. 


\section{Conclusions and Outlook}

In general, thanks to the high precision of single-ion Penning trap techniques, atomic mass measurements do not currently limit the determination of other fundamental constants. However, one can foresee instances where improvement will be needed for this to remain true. For light ions, if it becomes clear that muonic lamb-shift measurements are a valid method for measuring the proton and deuteron charge radii, atomic masses of the proton and deuteron relative to the electron at the sub-10-11 level will be needed to match the precision of current 1s-2s hydrogen spectroscopy. They will also be necessary for interpreting the results of precision ro-vibrational laser spectroscopy of $\mathrm{H}_{2}{ }^{+}$and $\mathrm{HD}^{+}$[65]. Regarding the mass of ${ }^{3} \mathrm{He}$, despite the 4-sigma disagreement, which should be resolved, the precision measurements of FSU [32,61] and UW [77] have reduced its uncertainty to the $1 \times 10^{-10}$ level, which should be adequate for an initial determination of the nuclear $g$-factor of ${ }^{3} \mathrm{He}$ [71]. Because of the importance of the $\mathrm{T}^{-}{ }^{3} \mathrm{He}$ mass difference for neutrino mass, more measurements of this difference are desirable. And, if a measurement of the $g$-factor of ${ }^{4} \mathrm{He}$ were to be used to obtain a value for the electron mass at the sub- $10^{-11}$ level of precision, a correspondingly precise value for $M\left[{ }^{4} \mathrm{He}\right]$ will be required. And of course, because of the possibility of unknown or underestimated systematic errors, it is always metrologically useful for the most precise measurements to be tested by another measurement of comparable precision.

Likewise, although the atomic masses of ${ }^{87} \mathrm{Rb}$ and ${ }^{133} \mathrm{Cs}$ are not yet a limit for obtaining the fine-structure constant using the atom-recoil method, this could change in the future. It would also be preferable for their masses to be referenced more directly to that of ${ }^{12} \mathrm{C}$ than is the case at present. In fact, to reduce the atomic mass uncertainty of a heavy atom like ${ }^{133} \mathrm{Cs}$ to the $10^{-11}$ level is challenging since forming an $\mathrm{m} / \mathrm{q}$ doublet would involve a large hydro-carbon molecule-with uncertain binding energy and polarizability corrections-or else a highly-charged Cs ion, also with uncertainty in the ionization energies. It is also worth noting that for obtaining $\alpha$ using Equation (4), it is not necessary for ${ }^{12} \mathrm{C}$ to be used as an intermediary. For example, an electronic $g$-factor measurement on ${ }^{4} \mathrm{He}^{+}$, say, could be combined with a measurement of the mass ratio of ${ }^{4} \mathrm{He}$ and ${ }^{133} \mathrm{Cs}$. And, as has been noted, high precision atom-recoil measurements are being developed in other systems such as ${ }^{7} \mathrm{Li}$ and isotopes of $\mathrm{Yb}$. Therefore, more precise mass measurements may become motivated for these atoms.

Funding: This work was partly supported by the National Science Foundation under NSF 1403725.

Acknowledgments: The author acknowledges D.J. Fink for reading the manuscript.

Conflicts of Interest: The author declares no conflict of interest.

\section{References}

1. Safranova, M.S.; Budker, D.; DeMille, D.; Jackson Kimball, D.F.; Derevianko, A.; Clark, C.W. Search for new physics with atoms and molecules. Rev. Mod. Phys. 2018, 90, 025008. [CrossRef]

2. Mohr, P.J.; Newell, D.B.; Taylor, B.N. CODATA recommended values of the fundamental physical constants 2014. Rev. Mod. Phys. 2016, 88, 035009. [CrossRef]

3. Mohr, P.J.; Newell, D.B.; Taylor, B.N.; Tiesinga, E. Data and analysis for the CODATA 2017 special fundamental constants adjustment. Metrologia 2018, 55, 125. [CrossRef]

4. Huang, W.J.; Audi, G.; Wang, M.; Kondev, F.G.; Naimi, S.; Xu, X. The AME2016 atomic mass evaluation (I) Evaluation of input data; and adjustment procedures. Chin. Phys. C 2017, 41, 030002. [CrossRef]

5. Wang, M.; Audi, G.; Kondev, F.G.; Huang, W.J.; Naimi, S.; Xu, X. The AME2016 atomic mass evaluation (II) Tables, graphs and references. Chin. Phys. C 2017, 41, 030003. [CrossRef]

6. Myers, E.G. The most precise atomic mass measurements in Penning traps. Int. J. Mass Spectrom. 2013, 349-350, 107. [CrossRef]

7. Blaum, K. High-accuracy mass spectrometry with stored ions. Phys. Rep. 2006, 425, 1. [CrossRef]

8. Kluge, H.-J. High-accuracy mass spectrometry for fundamental studies. Eur. J. Mass Spectrom. 2010, 16, 269. [CrossRef] 
9. Blaum, K.; Novikov, Y.N.; Werth, G. Penning traps as a versatile tool for precise experiments in fundamental physics. Contemp. Phys. 2010, 51, 149. [CrossRef]

10. Dilling, J.; Blaum, K.; Brodeur, M.; Eliseev, S. Penning-trap mass measurements in atomic and nuclear physics. Ann. Rev. Nucl. Part. Sci. 2018, 68, 45. [CrossRef]

11. Mohr, P.J.; Taylor, B.N. CODATA recommended values of the fundamental physical constants 2002. Rev. Mod. Phys. 2005, 77, 1. [CrossRef]

12. The UMIST Database for Astrochemistry. Available online: udfa.ajmarkwick.net/index.php (accessed on 29 December 2018).

13. Robinson, A.; Schlamminger, S. The watt or Kibble balance: A technique for implementing the new SI definition of the unit of mass. Metrologia 2016, 53, A46. [CrossRef]

14. Kuramoto, N.; Mizushima, S.; Zhang, L.; Fujita, K.; Azuma, Y.; Kurokawa, A.; Okubo, S.; Inaba, H.; Fujii, K. Determination of the Avogadro constant by the XRCD method using a ${ }^{28}$ Si-enriched sphere. Metrologia 2017, 54, 716. [CrossRef]

15. Redshaw, M.; McDaniel, J.; Myers, E.G. Dipole moment of $\mathrm{PH}^{+}$and the atomic masses of ${ }^{28} \mathrm{Si},{ }^{31} \mathrm{P}$ by comparing cyclotron frequencies of two ions simultaneously trapped in a Penning trap. Phys. Rev. Lett. 2008, 100, 093002. [CrossRef]

16. Lan, S.Y.; Kuan, P.-C.; Estey, B.; English, D.; Brown, J.M.; Hohensee, M.A.; Müller, H. A clock directly linking time to a particle's mass. Science 2013, 339, 554. [CrossRef]

17. Sturm, S.; Kohler, F.; Zatorski, J.; Wagner, A.; Harman, Z.; Werth, G.; Quint, W.; Keitel, C.H.; Blaum, K. High-precision measurement of the atomic mass of the electron. Nature 2014, 506, 467. [CrossRef]

18. Brown, L.S.; Gabrielse, G. Geonium theory: Physics of a single electron or ion in a Penning trap. Rev. Mod Phys. 1986, 58, 233. [CrossRef]

19. Sellner, S.; Besirli, M.; Bohman, M.; Borchert, M.J.; Harrington, J.; Higuchi1, T.; Mooser, A.; Nagahama, H.; Schneider, G.; Smorra, C.; et al. Improved limit on the directly measured antiproton lifetime. N. J. Phys. 2017, 19, 083023. [CrossRef]

20. Nagahama, H.; Schneider, G.; Mooser, A.; Smorra, C.; Sellner, S.; Harrington, J.; Higuchi, T.; Borchert, M.; Tanaka, T.; Bersirli, M.; et al. Highly sensitive superconducting circuits at $\sim 700 \mathrm{kHz}$ with tunable quality factors for image-current detection of single trapped antiprotons. Rev. Sci. Instrum. 2016, 87, 113305. [CrossRef]

21. Bradley, M.P. A Sub-Ppb Measurement of the Mass of Cesium for A New Determination of the Fine-Structure Constant. Ph.D. Thesis, Massachusetts Institute of Technology, Cambridge, MA, USA, 2000.

22. Gabrielse, G.; Khabbaz, A.; Hall, D.S.; Heimann, C.; Kalinowsky, H.; Jhe, W. Precision spectroscopy of the antiproton and proton using simultaneously trapped particles. Phys. Rev. Lett. 1999, 82, 3198. [CrossRef]

23. Ulmer, S.; Blaum, K.; Kracke, H.; Mooser, A.; Quint, W.; Rodegheri, C.C.; Walz, J. A cryogenic detection system at $28.9 \mathrm{MHz}$ for the non-destructive observation of a single proton at low particle energy. Nucl. Instrum. Method A 2013, 705, 55. [CrossRef]

24. Pinegar, D.B. Tools for a Precise Tritium to Helium-3 Mass Comparison. Ph.D. Thesis, University of Washington, Seattle, WA, USA, 2007.

25. Höcker, M. Precision Mass Measurements at THe Trap and the FSU Trap. Ph.D. Thesis, Ruprechts-KarlsUniversität Heidelberg, Heidelberg, Germany, 2016.

26. Cornell, E.A.; Weisskoff, R.M.; Boyce, K.R.; Pritchard, D.E. Mode coupling in a Penning trap: Pi pulses and a classical avoided crossing. Phys. Rev. A 1990, 41, 312. [CrossRef] [PubMed]

27. Ulmer, S.; Smorra, C.; Mooser, A.; Franke, K.; Nagahama, H.; Schneider, G.; Higuchi, T.; Van Gorp, S.; Blaum, K.; Matsuda, Y.; et al. High-precision comparison of the antiproton-to-proton charge to mass ratio. Nature 2015, 524, 196. [CrossRef] [PubMed]

28. Cornell, E.A.; Weisskoff, R.M.; Boyce, K.R.; Flanagan, R.W.; Lafyatis, G.P.; Pritchard, D.E. Single-ion cyclotron resonance measurement of $\mathrm{M}(\mathrm{CO}+) / \mathrm{M}(\mathrm{N} 2+)$. Phys. Rev. Lett. 1989, 63, 1674. [CrossRef] [PubMed]

29. Shi, W.; Redshaw, M.; Myers, E.G. Atomic masses of ${ }^{32,33} \mathrm{~S},{ }^{84,86} \mathrm{Kr}$, and ${ }^{129,132}$ Xe with uncertainties $<0.1 \mathrm{ppb}$. Phys. Rev. A 2005, 72, 022510. [CrossRef]

30. Redshaw, M.; Mount, B.J.; Myers, E.G. Improved masses of ${ }^{84,86} \mathrm{Kr}$ and ${ }^{129,132}$ Xe. Phys. Rev. A 2009, 79, 012506. [CrossRef]

31. Mount, B.J.; Redshaw, M.; Myers, E.G. Atomic masses of ${ }^{6} \mathrm{Li},{ }^{23} \mathrm{Na},{ }^{39,41} \mathrm{~K},{ }^{85,87} \mathrm{Rb}$, and ${ }^{133}$ Cs. Phys. Rev. A 2010, 82, 042513. [CrossRef] 
32. Myers, E.G.; Wagner, A.; Kracke, H.; Wesson, B.A. Atomic masses of tritium and helium-3. Phys. Rev. Lett. 2015, 114, 013003. [CrossRef]

33. Sturm, S.; Wagner, A.; Schabinger, B.; Blaum, K. Phase sensitive cyclotron frequency measurements at ultralow energies. Phys. Rev. Lett. 2011, 107, 143003. [CrossRef] [PubMed]

34. Bergström, I.; Carlberg, C.; Fritioff, T.; Douysset, G.; Schönfelder, J.; Schuch, R. SMILETRAP-A Penning trap facility for precision mass measurements using highly-charged ions. Nucl. Instrum. Method A 2002, 487, 618. [CrossRef]

35. Gabrielse, G. Why is sideband mass spectrometry possible with ions in a Penning trap? Phys. Rev. Lett. 2009, 102, 172501. [CrossRef]

36. Kretzschmar, M. The Ramsey method in high-precision mass spectrometry with Penning traps: Theoretical foundations. Int. J. Mass Spectrom. 2007, 264, 122. [CrossRef]

37. George, S.; Blaum, K.; Herfurth, F.; Herlert, A.; Kretzschmar, N.; Nagy, S.; Schwarz, S.; Schweikhard, L.; Yazidijian, C. The Ramsey method in high-precision mass spectrometry with Penning traps: Experimental results. Int. J. Mass Spectrom. 2007, 264, 110-121. [CrossRef]

38. Solders, A.; Bergström, I.; Nagy, S.; Suhonen, M.; Schuch, R. Determination of the proton mass from a measurement of the cyclotron frequencies of $\mathrm{D}^{+}$and $\mathrm{H}_{2}{ }^{+}$in a Penning trap. Phys. Rev. A 2008, 78, 012514. [CrossRef]

39. Eliseev, S.; Blaum, K.; Block, M.; Droese, C.; Goncharov, M.; Minaya Ramirez, E.; Nesterenko, D.A.; Novikov, Y.N.; Schweikhard, L. Phase-imaging ion-cyclotron-resonance measurements for short lived nuclides. Phys. Rev. Lett. 2013, 110, 082501. [CrossRef] [PubMed]

40. Van Dyck, R.S.; Zafonte, S.L.; Schwinberg, P.B. Ultra-precise mass measurements using the UW-PTMS. Hyperfine Interact. 2001, 132, 163. [CrossRef]

41. DiFilippo, F.; Natarajan, V.; Boyce, K.R.; Pritchard, D.E. Accurate atomic masses for fundamental metrology. Phys. Rev. Lett. 1994, 73, 1481. [CrossRef]

42. Redshaw, M.; McDaniel, J.; Shi, W.; Myers, E.G. Mass ratio of two ions in a Penning trap by alternating between the trap center and a large cyclotron orbit. Int. J. Mass Spectrom. 2006, 251, 125. [CrossRef]

43. Rainville, S.; Thompson, J.K.; Pritchard, D.E. An ion balance for ultra-high-precision atomic mass measurements. Science 2004, 303, 334. [CrossRef]

44. Repp, J.; Böhm, C.; López-Urrutia, J.R.C.; Dörr, A.; Eliseev, S.; George, S.; Goncharov, M.; Novikov, Y.N.; Roux, C.; Sturm, S.; et al. PENTATRAP: A novel cryogenic multi-Penning-trap experiment for high-precision mass measurements on highly charged ions. Appl. Phys. B 2012, 107, 983. [CrossRef]

45. Schüssler, R.X.; Door, M.; Rischka, A.; Bekker, H.; Crespo López-Urrutia, J.R.; Filianin, P.; Eliseev, S.; Novikov, Y.N.; Sturm, S.; Ulmer, S.; et al. Recent developments at the high-precision mass spectrometer PENTATRAP. JPS Conf. Proc. 2017, 18, 011020.

46. Heisse, F.; Köhler-Langes, F.; Rau, S.; Hou, J.; Junck, S.; Kracke, A.; Mooser, A.; Quint, W.; Ulmer, S.; Werth, G.; et al. High-precision measurement of the proton's atomic mass. Phys. Rev. Lett. 2017, 119, 033001. [CrossRef] [PubMed]

47. Redshaw, M.; Bryce, R.A.; Hawks, P.; Gamage, N.D.; Hunt, C.; Kandegedara, R.M.E.B.; Rantnayake, I.S.; Sharp, L. Status and outlook of CHIP-TRAP: The Central Michigan University high-precision Penning trap. Nucl. Instrum. Method B 2016, 376, 302. [CrossRef]

48. Rainville, S. A Two-Ion Balance for High Precision Mass Spectrometry. Ph.D. Thesis, Massachusetts Institute of Technology, Cambridge, MA, USA, 2003.

49. Ketter, J.; Eronen, T.; Höcker, M.; Streubel, S.; Blaum, K. First-order perturbative calculations of the frequency-shifts caused by static cylindrically-symmetric electric and magnetic imperfections of a Penning trap. Int. J. Mass Spectrom. 2014, 358, 1. [CrossRef]

50. Ketter, J.; Eronen, T.; Höcker, M.; Schuh, M.; Streubel, S.; Blaum, K. Classical calculations of relativistic frequency-shifts in an ideal Penning trap. Int. J. Mass Spectrom. 2014, 361, 34. [CrossRef]

51. Porto, J.V. Series solution for the image charge fields in arbitrary cylindrically symmetric Penning traps. Phys. Rev. A 2001, 64, 023403. [CrossRef]

52. Thompson, J.K. Two Ion Control and Polarization Forces for Precise Mass Comparisons. Ph.D. Thesis, Massachusetts Institute of Technology, Cambridge, MA, USA, 2003.

53. Sturm, S. The $g$-Factor of the Bound Electron in ${ }^{28} \mathrm{Si}^{13+}$, the Most Stringent Test of Bound-State Quantum Electrodynamics. Ph.D. Thesis, Johannes-Gutenberg Universität Mainz, Mainz, Germany, 2012. 
54. Guise, N.; DiSciacca, J.; Gabrielse, G. Self-excitation and feedback cooling of an isolated proton. Phys. Rev. Lett. 2010, 104, 143001. [CrossRef] [PubMed]

55. Fink, D.; Smith, J.; Hamzeloui, S.; Myers, E. Precision mass measurements with molecular ions: Resolving rotational and vibrational energy. Bull. Am. Phys. Soc. 2018, 65, 193.

56. Smith, J.A.; Hamzeloui, S.; Fink, D.J.; Myers, E.G. Rotational energy as mass in $\mathrm{H}_{3}{ }^{+}$and lower limits on the atomic masses of D an ${ }^{3}$ He. Phys. Rev. Lett. 2018, 120, 143002. [CrossRef] [PubMed]

57. Thompson, J.K.; Rainville, S.; Pritchard, D.E. Cyclotron frequency shifts arising from polarization forces. Nature 2004, 430, 58. [CrossRef]

58. Cheng, M.; Brown, J.M.; Rosmus, P.; Linguerri, R.; Komiha, N.; Myers, E.G. Dipole moments and orientation polarizabilities of diatomic molecular ions for precision atomic mass measurement. Phys. Rev. A 2007, 75, 012502. [CrossRef]

59. Rainville, S.; Thompson, J.K.; Myers, E.G.; Brown, J.M.; Dewey, M.S.; Kessler, E.G.; Deslattes, R.D.; Börner, H.G.; Jentschel, M.; Mutti, P.; et al. A direct test of $E=m c^{2}$. Nature 2005, 438, 1096. [CrossRef] [PubMed]

60. Mount, B.J.; Redshaw, M.; Myers, E.G. Dipole moments of $\mathrm{HCO}^{+}$and $\mathrm{NH}^{+}$from cyclotron frequency polarizability shifts. Phys. Rev. A 2012, 85, 012519. [CrossRef]

61. Hamzeloui, S.; Smith, J.A.; Fink, D.J.; Myers, E.G. Precision mass ratio of ${ }^{3} \mathrm{He}^{+}$to $\mathrm{HD}^{+}$. Phys. Rev. A 2017, 96, 060501.

62. Parthey, C.G.; Matveev, A.; Alnis, J.; Bernhardt, B.; Beyer, A.; Holzwarth, R.; Maistrou, A.; Pohl, R.; Predehl, K.; Udem, T.; et al. Improved measurement of the hydrogen 1s-2s transition frequency. Phys. Rev. Lett. 2011, 107, 203001. [CrossRef] [PubMed]

63. Pohl, R.; Antognini, A.; Kottmann, F. The size of the proton. Nature 2010, 466, 213. [CrossRef]

64. Hilico, L.; Billy, N.; Grémaud, B.; Delande, D. Ab initio calculations of the $\mathrm{J}=0$ to $\mathrm{J}=1$ states of the $\mathrm{H}_{2}{ }^{+}, \mathrm{D}_{2}{ }^{+}$ and $\mathrm{HD}^{+}$molecular ions. Eur. Phys. J. D 2000, 12, 449. [CrossRef]

65. Korobov, V.I.; Hilico, L.; Karr, J.-P. Theoretical transitions frequencies beyond $0.1 \mathrm{ppb}$ accuracy in $\mathrm{H}_{2}{ }^{+}, \mathrm{HD}^{+}$ and antiprotonic helium. Phys. Rev. A 2014, 89, 032511. [CrossRef]

66. Biesheuvel, J.; Karr, J.-P.; Hilico, L.; Eikema, K.S.E.; Ubachs, W.; Koelemeij, J.C.J. High-precision spectroscopy of the $\mathrm{HD}^{+}$molecule at the 1 ppb level. Appl. Phys. B 2017, 123, 1. [CrossRef]

67. Karr, J.-P.; Hilico, L.; Koelemeij, J.C.J.; Korobov, V.I. Hydrogen molecular ions for improved determination of fundamental constants. Phys. Rev. A 2016, 94, 050501. [CrossRef]

68. Myers, E.G. CPT tests with the antihydrogen molecular ion. Phys. Rev. A 2018, 98, 010101. [CrossRef]

69. Otten, E.W.; Bonn, J.; Weinheimer, C. The Q-value of tritium-beta decay and the neutrino mass. Int. J. Mass Spectrom. 2006, 251, 173. [CrossRef]

70. Arenz, M.; Baek, W.J.; Beck, M.; Beglarian, A.; Behrens, J.; Bergmann, T.; Berlev, A.; Besserer, U.; Blaum, K.; Bodeet, T.; et al. Calibration of high voltages at the ppm level by the differences of ${ }^{83 \mathrm{~m}} \mathrm{Kr}$ conversion electron lines at the KATRIN experiment. Eur. Phys. J. C 2018, 78, 368. [CrossRef]

71. Mooser, A.; Rischka, A.; Schneider, A.; Blaum, K.; Ulmer, S.; Walz, J. A new experiment for the measurement of the $g$-factors of ${ }^{3} \mathrm{He}^{+}$and ${ }^{3} \mathrm{He}^{2+}$. J. Phys. Conf. Ser. 2018, 1138, 012004. [CrossRef]

72. Jentschura, U.D.; Czarnecki, A.; Pachucki, K.; Yerokhin, V.A. Mass measurements and the bound-electron g-factor. Int. J. Mass Spectrom. 2006, 251, 102. [CrossRef]

73. Natarajan, V.; Boyce, K.R.; DiFilippo, F.; Pritchard, D.E. Precision Penning trap comparisons of nondoublets: Atomic masses of H, D and the neutron. Phys. Rev. Lett. 1993, 71, 1998. [CrossRef]

74. Van Dyck, R.S.; Farnham, D.L.; Zafonte, S.L.; Schwinberg, P.B. High precision Penning trap mass spectroscopy and a new measurement of the proton's atomic mass. AIP Conf. Proc. 1999, 457, 101-110.

75. Bergström, I.; Fritioff, T.; Schuch, R.; Schönfelder, J. On the masses of ${ }^{28} \mathrm{Si}$ and the proton determined in a Penning trap. Phys. Scr. 2002, 66, 201. [CrossRef]

76. Karr, J.-P. Stark-quenching of rovibrational states of $\mathrm{H}_{2}{ }^{+}$due to motion in a magnetic field. Phys. Rev. A 2018, 98, 062501. [CrossRef]

77. Zafonte, S.L.; Van Dyck, R.S. Ultra-precise single-ion atomic mass measurements on deuterium and helium-3. Metrologia 2015, 52, 280. [CrossRef]

78. Kessler, E.G.; Dewey, M.S.; Deslattes, R.D.; Henins, A.; Börner, H.G.; Jentschel, M.; Doll, C.; Lehmann, H. The deuteron binding energy and the neutron mass. Phys. Lett. A 1999, 255, 221. [CrossRef] 
79. Nagy, S.; Fritioff, T.; Björkhage, M.; Bergström, I.; Schuch, R. On the $Q$-value of tritium beta-decay. Europhys. Lett. 2006, 74, 404. [CrossRef]

80. Mohr, P.J.; Taylor, B.N.; Newell, D.B. CODATA recommended values of the fundamental physical constants 2010. Rev. Mod. Phys. 2012, 48, 1527. [CrossRef]

81. Yan, Z.-C.; Zhang, J.-Y.; Li, Y. Energies and polarizabilities of the hydrogen molecular ions. Phys. Rev. A 2003, 67, 062504. [CrossRef]

82. Van Dyck, R.S.; Farnham, D.L.; Schwinberg, P.B. Tritium-helium-3 mass difference using the Penning trap mass spectroscopy. Phys. Rev. Lett. 1993, 70, 2888. [CrossRef] [PubMed]

83. Van Dyck, R.S.; Zafonte, S.L.; Van Liew, S.; Pinegar, D.B.; Schwinberg, P.B. Ultra precise atomic mass measurement of the $\alpha$ particle and ${ }^{4} \mathrm{He}$. Phys. Rev. Lett. 2004, 92, 220802. [CrossRef] [PubMed]

84. Brunner, S.; Engel, T.; Schmitt, A.; Werth, G. Determination of the helium-4 mass in a Penning trap. Eur. Phys. J. D 2001, 15, 181. [CrossRef]

85. Fritioff, T.; Carlberg, C.; Douysset, G.; Schuch, R.; Bergström, I. A new determination of the ${ }^{4} \mathrm{He}$ and ${ }^{3} \mathrm{He}$ masses in a Penning trap. Eur. Phys. J. D 2001, 15, 141. [CrossRef]

86. Aoyama, T.; Kinoshita, T.; Nio, M. Revised and improved value of the QED tenth-order electron anomalous magnetic moment. Phys. Rev. D 2018, 97, 036001. [CrossRef]

87. Hanneke, D.; Fogwell, S.; Gabrielse, G. New measurement of the electron magnetic moment and the fine-structure constant. Phys. Rev. Lett. 2008, 100, 120801. [CrossRef]

88. Bouchendira, R.; Cladé, P.; Guellati-Khélifa, S.; Nez, F.; Biraben, F. New determination of the fine structure constant and test of quantum electrodynamics. Phys. Rev. Lett. 2011, 106, 080801. [CrossRef] [PubMed]

89. Parker, R.H.; Yu, C.; Zhong, W.; Estey, B.; Müller, H. Measurement of the fine-structure constant as a test of the standard model. Science 2018, 360, 191. [CrossRef] [PubMed]

90. Carlberg, C.; Fritioff, T.; Bergström, I. Determination of the ${ }^{133} \mathrm{Cs}$ and proton mass ratio using highly charged ions. Phys. Rev. Lett. 1999, 83, 4506. [CrossRef]

91. Bradley, M.P.; Porto, J.V.; Rainville, S.; Thompson, J.K.; Pritchard, D.E. Penning trap measurements of the masses of ${ }^{133} \mathrm{Cs},{ }^{85,87} \mathrm{Rb}$, and ${ }^{23} \mathrm{Na}$ with uncertainties $\leq 0.2 \mathrm{ppb}$. Phys. Rev. Lett. 1999, 83, 4510.

92. Audi, G.; Wapstra, A.H.; Thibault, C. The AME2003 atomic mass evaluation (II). Tables, graphs and references. Nucl. Phys. A 2003, 729, 337. [CrossRef]

93. Cassella, K.; Copenhaver, E.; Estey, B.; Feng, Y.; Lai, C.; Müller, H. Recoil-sensitive lithium interferometer without a sub-recoil sample. Phys. Rev. Lett. 2017, 118, 233201. [CrossRef]

94. Zhang, X.; del Aguila, R.P.; Mazzoni, T.; Poli, N.; Tino, G.M. Trapped-atom interferometer with ultracold Sr atoms. Phys. Rev. A 2016, 94, 043608. [CrossRef]

95. Jamison, A.O.; Plotkin-Swing, B.; Gupta, S. Advances in precision contrast interferometry with $\mathrm{Yb}$ Bose-Einstein condensates. Phys. Rev. A 2014, 90, 063606. [CrossRef]

96. Rana, R.; Höcker, M.; Myers, E.G. Atomic masses of strontium and ytterbium. Phys. Rev. A 2012, 86, 050502(R). [CrossRef]

97. Farnham, D.L.; Van Dyck, R.S.; Schwinberg, P.B. Determination of the electron's atomic mass and the proton/electron mass ratio via Penning trap mass spectrometry. Phys. Rev. Lett. 1995, 75, 3598. [CrossRef]

98. Beier, T.; Häffner, H.; Hermanspahn, N.; Karshenboim, S.G.; Kluge, H.J.; Quint, W.; Stahl, S.; Verdú, J.; Werth, G. New determination of the electron's mass. Phys. Rev. Lett. 2001, 88, 011603. [CrossRef] [PubMed]

99. Verdú, J.; Djekić, S.; Stahl, S.; Valenzuela, T.; Vogel, M.; Werth, G.; Beier, T.; Kluge, H.J.; Quint, W. Electronic g-factor of hydrogenlike oxygen ${ }^{16} \mathrm{O}^{7+}$. Phys. Rev. Lett. 2004, 92, 093002. [CrossRef]

100. Köhler, F.; Sturm, S.; Kracke, A.; Werth, G.; Quint, W.; Blaum, K. The electron mass from $g$-factor measurements on hydrogen-like carbon ${ }^{12} \mathrm{C}^{5+}$. J. Phys. B. At. Mol. Opt. Phys. 2015, 48, 144032. [CrossRef]

101. Zatorski, J.; Sikora, B.; Karshenboim, S.G.; Sturm, S.; Köhler-Langes, F.; Blaum, K.; Keitel, C.H.; Harman, Z. Extraction of the electron mass from g-factor measurements of light hydrogenlike ions. Phys. Rev. A 2017, 96, 012502. [CrossRef]

102. Pachucki, K.; Czarnecki, A.; Jentschura, U.D.; Yerokhin, V.A. Complete two-loop correction to the bound-electron g-factor. Phys. Rev. A 2005, 72, 022108. [CrossRef]

103. Sturm, S.; Vogel, M.; Köhler-Langes, F.; Quint, W.; Blaum, K.; Werth, G. High-precision measurements of the bound electron's magnetic moment. Atoms 2017, 5, 4. [CrossRef] 
104. Sturm, S.; Wagner, A.; Kretzschmar, M.; Quint, W.; Werth, G.; Blaum, K. g-factor measurement of hydrogenlike ${ }^{28} \mathrm{Si}^{13+}$ as a challenge to QED calculations. Phys. Rev. A 2013, 87, 030501. [CrossRef]

105. Shabaev, V.M.; Glazov, D.A.; Plunien, G.; Volotka, A.V. Theory of bound-electron $g$-factor in highly charged ions. J. Phys. Chem. Ref. Data 2015, 44, 031205. [CrossRef] 